\title{
A posteriori error analysis of a fully-mixed formulation for the Brinkman-Darcy problem
}

\author{
M. Álvarez ${ }^{1}$ - G. N. Gatica ${ }^{2}$ - R. Ruiz-Baier ${ }^{3}$
}

Received: 24 June 2017 / Accepted: 29 August 2017 / Published online: 5 September 2017

(C) The Author(s) 2017. This article is an open access publication

\begin{abstract}
We develop the a posteriori error analysis for a mixed finite element method applied to the coupling of Brinkman and Darcy equations in 3D, modelling the interaction of viscous and non-viscous flow effects across a given interface. The system is formulated in terms of velocity and pressure within the Darcy subdomain, together with vorticity, velocity and pressure of the fluid in the Brinkman region, and a Lagrange multiplier enforcing pressure continuity across the interface. The solvability of a fullymixed formulation along with a priori error bounds for a finite element method have been recently established in Álvarez et al. ( Comput Methods Appl Mech Eng 307:68$95,2016)$. Here we derive a residual-based a posteriori error estimator for such a scheme, and prove its reliability exploiting a global inf-sup condition in combination with suitable Helmholtz decompositions, and interpolation properties of Clément and Raviart-Thomas operators. The estimator is also shown to be efficient, following a localisation strategy and appropriate inverse inequalities. We present numerical tests to confirm the features of the estimator and to illustrate the performance of the method in academic and application-oriented problems.
\end{abstract}

$凶$ R. Ruiz-Baier

ruizbaier@maths.ox.ac.uk

M. Álvarez

mario.alvarezguadamuz@ucr.ac.cr

G. N. Gatica

ggatica@ci2ma.udec.cl

1 Sección de Matemática, Sede de Occidente, Universidad de Costa Rica, San Ramón de Alajuela, Costa Rica

$2 \mathrm{CI}^{2} \mathrm{MA}$ and Departamento de Ingeniería Matemática, Universidad de Concepción, Casilla 160-C, Concepción, Chile

3 Mathematical Institute, Oxford University, A. Wiles Building, Radcliffe Observatory Quarter, Woodstock Road, Oxford OX2 6GG, UK 
Keywords Brinkman-Darcy equations · Vorticity-based formulation · Mixed finite element methods · A posteriori error analysis

Mathematics Subject Classification 65 N30 $\cdot 65 \mathrm{~N} 12 \cdot 76 \mathrm{D} 07 \cdot 65 \mathrm{~N} 15$

\section{Introduction}

We have recently introduced a mixed finite element method to numerically approximate the flow patterns of a viscous fluid within a highly permeable medium described by Brinkman equations, and its interaction with pure porous media flow under Darcy's law [1]. There, the system is formulated in terms of velocity and pressure of the nonviscous flow, together with vorticity, velocity and pressure of the Brinkman region. The tangential vorticity vanishes on the boundary of the Brinkman domain, whereas slip velocity conditions are assumed on the overall boundary. The corresponding mixed variational formulation leads to a Lagrange multiplier enforcing pressure continuity across the interface, while mass balance results from essential boundary conditions on each domain. As a consequence, a classical saddle-point operator equation is obtained, whose invertibility hinges on the well-known Babuška-Brezzi theory. A similar treatment is used to establish the solvability of the discrete problem associated to the Galerkin method. The needed continuous and discrete inf-sup conditions can be guaranteed thanks to the so-called $T$-coercivity argument (cf. $[18,26]$ and the references therein), where one defines adequate injective operators delivering lower bounds of the corresponding suprema. As the stability of the Galerkin scheme requires that the curl of the discrete vorticity space is contained in the discrete Brinkman velocity space, we specify Raviart-Thomas and Nédélec spaces for the approximation of the global velocity and the Brinkman vorticity, respectively.

On the other hand, the derivation of adaptive schemes for transmission free flow-porous media problems has been extensively studied in recent years. In particular, we refer to $[6,9-11,14,16,27]$, which are focused on Stokes-Darcy and Navier-Stokes/Darcy couplings, and where the interface conditions are treated in different ways, from both mathematical and numerical perspective. For instance, in $[6,14,16,27]$, Beavers-Joseph-Saffman-type conditions are considered on the interface, whereas in $[10,11]$, similar transmission conditions to those employed in [1] are assumed. Also, an interesting feature of the proof of reliability in [6], which differs from the approaches in the other works, is the utilisation of intermediate inf-sup inequalities that are obtained along the proof of the global inf-sup condition. Differently from the above, and similarly as in [14,16,27], the efficiency estimates in [6] follow from usual arguments based on inverse inequalities and the localisation technique employing triangle-bubble and edge-bubble functions. In turn, the assumption of a smallness condition on the data is the distinctive feature of the approach in [16], where a reliable and efficient residual-based a posteriori error estimator for the three dimensional version of the augmented-mixed method introduced in [17], is derived. Furthermore, an a posteriori error estimator for a conforming and nonconforming vorticity-based finite element method of a Stokes-Darcy coupled problem was derived in $[10,11]$, respectively, but the resulting estimate in [11] is not optimal. In addition, even though 
in $[10,11]$ the model problem is addressed for both two and three spatial dimensions, the corresponding a posteriori error analysis is explicitly derived only for the $2 \mathrm{D}$ case.

According to the previous discussion, and as a natural continuation of the a priori error analysis developed in [1], our goal in the present paper is to provide a reliable and efficient residual-based a posteriori error estimator for the finite element method introduced and analyzed in that reference. In this way, we aim to improve the accuracy of the discrete scheme from [1] in different scenarios, including presence of singularities or high gradients of the solution. Indeed, in contrast with the methodology developed in $[10,11]$, and following the approaches in $[16,27]$, we highlight that the derivation of our error estimator is based on a global inf-sup condition in combination with suitable Helmholtz decompositions adapted from [16,31], and local approximation properties of Clément, Raviart-Thomas, and Clément-type Nédélec interpolators. Then, similarly as in $[14,16,27]$, the associated efficiency estimates are consequence of suitable inverse inequalities and local bounds for tetrahedron-bubble and facet-bubble functions.

The remainder of the paper is structured in the following manner. General preliminary notation is presented in the last part of this section. The model problem and the mixed variational formulation are outlined in Sect. 2, where we also recall its unique solvability and the mixed finite element discretisation. The core of the present analysis is contained in Sect. 3, where we define the error estimator and provide a detailed derivation of its reliability and efficiency. Finally, Sect. 4 gives two numerical tests aimed to illustrate the features of the method and the proposed estimator.

Some recurrent notation to be employed throughout the paper includes the following. If $S \subseteq \mathbb{R}^{3}$ is a domain or a Lipschitz surface, and $r \in \mathbb{R}$, we set vectorial Sobolev spaces as $\mathbf{H}^{r}(S):=\left[\mathrm{H}^{r}(S)\right]^{3}$, adopt the convention $\mathbf{H}^{0}(S) \equiv \mathbf{L}^{2}(S)$, and denote the corresponding norms by $\|\cdot\|_{r, S}$ (for both $\mathrm{H}^{r}(S)$ and $\mathbf{H}^{r}(S)$ ). In general, given a generic Hilbert space $\mathrm{H}$, we will employ $\mathbf{H}$ to denote its vectorial counterpart $\mathrm{H}^{3}$. We also recall the definition of the Hilbert spaces

$$
\begin{aligned}
\mathbf{H}(\operatorname{div} ; S):=\left\{\boldsymbol{v} \in \mathbf{L}^{2}(S):\right. & \left.\operatorname{div} \boldsymbol{v} \in \mathrm{L}^{2}(S)\right\} \\
\mathbf{H}(\mathbf{c u r l} ; S):=\left\{\boldsymbol{v} \in \mathbf{L}^{2}(S):\right. & \left.\operatorname{curl} \boldsymbol{v} \in \mathbf{L}^{2}(S)\right\}
\end{aligned}
$$

normed, respectively, with

$$
\|\boldsymbol{v}\|_{\text {div } S}:=\left\{\|\boldsymbol{v}\|_{0, S}^{2}+\|\operatorname{div} \boldsymbol{v}\|_{0, S}^{2}\right\}^{1 / 2}, \quad\|\boldsymbol{v}\|_{\text {curl } ; S}:=\left\{\|\boldsymbol{v}\|_{0, S}^{2}+\|\operatorname{curl} \boldsymbol{v}\|_{0, S}^{2}\right\}^{1 / 2},
$$

where, for any vector field $\boldsymbol{v}:=\left(v_{1}, v_{2}, v_{3}\right)^{\mathrm{t}} \in \mathbf{L}^{2}(S)$ we have

$$
\operatorname{div} \boldsymbol{v}:=\sum_{i=1}^{3} \partial_{i} v_{i} \text { and } \operatorname{curl} \boldsymbol{v}:=\nabla \times \boldsymbol{v}=\left(\begin{array}{l}
\partial_{2} v_{3}-\partial_{3} v_{2} \\
\partial_{3} v_{1}-\partial_{1} v_{3} \\
\partial_{1} v_{2}-\partial_{2} v_{1}
\end{array}\right)
$$

In addition we will use the space

$$
\mathrm{L}_{0}^{2}(S):=\left\{q \in \mathrm{L}^{2}(S): \int_{S} q=0\right\}
$$


endowed with the the usual norm of $\mathrm{L}^{2}(S)$. In turn, for each integer $k \geq 0$ we denote by $P_{k}(S)$ the space of polynomials in $S$ of total degree $\leq k$, and we set $\mathbf{P}_{k}(S)=\left[P_{k}(S)\right]^{3}$. Finally, the symbol $\mathbf{0}$ will stand for a generic null vector (including the null functional and operator), and $C$ (indistinguishably $c$, with or without subscripts, bars, tildes or hats) will denote generic constants independent of the discretisation parameters.

\section{Governing equations and a mixed variational formulation}

\subsection{The continuous model}

We first let $\Omega_{\mathrm{B}}$ and $\Omega_{\mathrm{D}}$ be bounded and simply connected polyhedral Lipschitz domains in $\mathbb{R}^{3}$ such that $\partial \Omega_{\mathrm{B}} \cap \partial \Omega_{\mathrm{D}}=: \Sigma \neq \emptyset$ and $\Omega_{\mathrm{B}} \cap \Omega_{\mathrm{D}}=\emptyset$, and set $\Omega:=\Omega_{\mathrm{B}} \cup \Sigma \cup \Omega_{\mathrm{D}}$ with boundary $\Gamma=\partial \Omega$ split into $\Gamma_{\mathrm{B}} \subseteq \partial \Omega_{\mathrm{B}}$ and $\Gamma_{\mathrm{D}} \subseteq \partial \Omega_{\mathrm{D}}$. Then, given source terms $f_{\mathrm{D}} \in \mathbf{L}^{2}\left(\Omega_{\mathrm{D}}\right)$ and $\boldsymbol{f}_{\mathrm{B}} \in \mathbf{L}^{2}\left(\Omega_{\mathrm{B}}\right)$, we are interested in the Brinkman-Darcy coupled problem

$$
\begin{aligned}
& \kappa_{\mathrm{B}}^{-1} \boldsymbol{u}_{\mathrm{B}}+v \operatorname{curl} \boldsymbol{\omega}_{\mathrm{B}}+\nabla p_{\mathrm{B}}=\boldsymbol{f}_{\mathrm{B}}, \quad \boldsymbol{\omega}_{\mathrm{B}}-\operatorname{curl} \boldsymbol{u}_{\mathrm{B}}=\mathbf{0}, \quad \operatorname{div} \boldsymbol{u}_{\mathrm{B}}=0 \text { in } \Omega_{\mathrm{B}}, \\
& \kappa_{\mathrm{D}}^{-1} \boldsymbol{u}_{\mathrm{D}}+\nabla p_{\mathrm{D}}=\boldsymbol{f}_{\mathrm{D}}, \quad \operatorname{div} \boldsymbol{u}_{\mathrm{D}}=0 \quad \text { in } \Omega_{\mathrm{D}}, \\
& \boldsymbol{\omega}_{\mathrm{B}} \times \boldsymbol{n}=\mathbf{0}, \quad \boldsymbol{u}_{\mathrm{D}} \cdot \boldsymbol{n}=\boldsymbol{u}_{\mathrm{B}} \cdot \boldsymbol{n}, \quad p_{\mathrm{D}}=p_{\mathrm{B}} \text { on } \Sigma, \\
& \boldsymbol{\omega}_{\mathrm{B}} \times \boldsymbol{n}=\mathbf{0}, \quad \boldsymbol{u}_{\mathrm{B}} \cdot \boldsymbol{n}=0 \text { on } \Gamma_{\mathrm{B}}, \\
& \boldsymbol{u}_{\mathrm{D}} \cdot \boldsymbol{n}=0 \text { on } \Gamma_{\mathrm{D}},
\end{aligned}
$$

which is formulated in terms of the Brinkman velocity $\boldsymbol{u}_{\mathrm{B}}$, the Brinkman pressure $p_{\mathrm{B}}$, the Brinkman vorticity $\omega_{\mathrm{B}}$, the Darcy velocity $\boldsymbol{u}_{\mathrm{D}}$, and the Darcy pressure $p_{\mathrm{D}}$. Here $\boldsymbol{n}$ stands for the outward normal at $\Omega_{\mathrm{B}}$ and $\Omega_{\mathrm{D}}, v>0$ is the kinematic viscosity of the fluid, and $\kappa_{\mathrm{D}}, \kappa_{\mathrm{B}}>0$ are the absolute permeabilities of the Darcy and Brinkman subdomains, respectively.

The boundary conditions on the Brinkman and Darcy subdomains suggest the following spaces

$$
\begin{aligned}
& \mathbf{H}_{\mathrm{B}}\left(\operatorname{div} ; \Omega_{\mathrm{B}}\right):=\left\{\boldsymbol{v}_{\mathrm{B}} \in \mathbf{H}\left(\operatorname{div} ; \Omega_{\mathrm{B}}\right): \quad \boldsymbol{v}_{\mathrm{B}} \cdot \boldsymbol{n}=0 \text { on } \Gamma_{\mathrm{B}}\right\}, \\
& \mathbf{H}_{\mathrm{D}}\left(\operatorname{div} ; \Omega_{\mathrm{D}}\right):=\left\{\boldsymbol{v}_{\mathrm{D}} \in \mathbf{H}\left(\operatorname{div} ; \Omega_{\mathrm{D}}\right): \boldsymbol{v}_{\mathrm{D}} \cdot \boldsymbol{n}=0 \text { on } \Gamma_{\mathrm{D}}\right\}, \\
& \mathbf{H}_{0}\left(\operatorname{curl} ; \Omega_{\mathrm{B}}\right):=\left\{z_{\mathrm{B}} \in \mathbf{H}\left(\operatorname{curl} ; \Omega_{\mathrm{B}}\right): \quad z_{\mathrm{B}} \times \boldsymbol{n}=\mathbf{0} \quad \text { on } \quad \partial \Omega_{\mathrm{B}}=\Gamma_{\mathrm{B}} \cup \Sigma\right\} .
\end{aligned}
$$

In addition, the pressure continuity across the interface $\Sigma$ allows us to define its trace via the auxiliary unknown $\lambda:=\left.p_{\mathrm{D}}\right|_{\Sigma}=\left.p_{\mathrm{B}}\right|_{\Sigma} \in \mathrm{H}^{1 / 2}(\Sigma)$, where $\langle\cdot, \cdot\rangle_{\Sigma}$ denotes the duality pairing of $\mathrm{H}^{-1 / 2}(\Sigma)$ and $\mathrm{H}^{1 / 2}(\Sigma)$ with respect to the $\mathrm{L}^{2}(\Sigma)$-inner product. In turn, the continuity of normal velocities across $\Sigma$ is imposed in a weak manner as

$$
\left\langle\boldsymbol{u}_{\mathrm{B}} \cdot \boldsymbol{n}-\boldsymbol{u}_{\mathrm{D}} \cdot \boldsymbol{n}, \xi\right\rangle_{\Sigma}=0 \quad \forall \xi \in \mathrm{H}^{1 / 2}(\Sigma)
$$


Then, a fully-mixed formulation for (2.1) reads as follows: Find $\boldsymbol{u}:=\left(\boldsymbol{u}_{\mathrm{B}}, \boldsymbol{\omega}_{\mathrm{B}}, \boldsymbol{u}_{\mathrm{D}}\right) \in$ $\mathbf{H}$ and $\mathbf{p}:=\left(p_{\mathrm{B}}, p_{\mathrm{D}}, \lambda\right) \in \mathbf{Q}_{0}$ such that

$$
\begin{aligned}
\mathbf{a}(\boldsymbol{u}, \boldsymbol{v})+\mathbf{b}(\boldsymbol{v}, \mathbf{p}) & =\mathbf{F}(\boldsymbol{v}) & & \forall \boldsymbol{v}:=\left(\boldsymbol{v}_{\mathrm{B}}, \boldsymbol{z}_{\mathrm{B}}, \boldsymbol{v}_{\mathrm{D}}\right) \in \mathbf{H}, \\
\mathbf{b}(\boldsymbol{u}, \mathbf{q}) & =\mathbf{0} & & \forall \mathbf{q}:=\left(q_{\mathrm{B}}, q_{\mathrm{D}}, \xi\right) \in \mathbf{Q}_{0}
\end{aligned}
$$

where $\mathbf{H}:=\mathbf{H}_{\mathrm{B}}\left(\operatorname{div} ; \Omega_{\mathrm{B}}\right) \times \mathbf{H}_{0}\left(\operatorname{curl} ; \Omega_{\mathrm{B}}\right) \times \mathbf{H}_{\mathrm{D}}\left(\operatorname{div} ; \Omega_{\mathrm{D}}\right), \mathbf{Q}_{0}:=\mathrm{L}_{0}^{2}\left(\Omega_{\mathrm{B}}\right) \times \mathrm{L}^{2}\left(\Omega_{\mathrm{D}}\right) \times$ $\mathrm{H}^{1 / 2}(\Sigma)$, and the bilinear forms $\mathbf{a}: \mathbf{H} \times \mathbf{H} \rightarrow \mathbb{R}$ and $\mathbf{b}: \mathbf{H} \times \mathbf{Q}_{0} \rightarrow \mathbb{R}$, and the functional $\mathbf{F} \in \mathbf{H}^{\prime}$, are defined by

$$
\begin{gathered}
\mathbf{a}(\boldsymbol{u}, \boldsymbol{v}):=\kappa_{\mathrm{B}}^{-1} \int_{\Omega_{\mathrm{B}}} \boldsymbol{u}_{\mathrm{B}} \cdot \boldsymbol{v}_{\mathrm{B}}+v \int_{\Omega_{\mathrm{B}}} \boldsymbol{\omega}_{\mathrm{B}} \cdot \boldsymbol{z}_{\mathrm{B}}+v \int_{\Omega_{\mathrm{B}}} \boldsymbol{v}_{\mathrm{B}} \cdot \operatorname{curl} \boldsymbol{\omega}_{\mathrm{B}} \\
-v \int_{\Omega_{\mathrm{B}}} \boldsymbol{u}_{\mathrm{B}} \cdot \operatorname{curl} z_{\mathrm{B}}+\kappa_{\mathrm{D}}^{-1} \int_{\Omega_{\mathrm{D}}} \boldsymbol{u}_{\mathrm{D}} \cdot \boldsymbol{v}_{\mathrm{D}}, \\
\mathbf{b}(\boldsymbol{v}, \mathbf{q}):=-\int_{\Omega_{\mathrm{B}}} q_{\mathrm{B}} \operatorname{div} \boldsymbol{v}_{\mathrm{B}}-\int_{\Omega_{\mathrm{D}}} q_{\mathrm{D}} \operatorname{div} \boldsymbol{v}_{\mathrm{D}}+\left\langle\boldsymbol{v}_{\mathrm{B}} \cdot \boldsymbol{n}-\boldsymbol{v}_{\mathrm{D}} \cdot \boldsymbol{n}, \xi\right\rangle_{\Sigma},
\end{gathered}
$$

and

$$
\mathbf{F}(\boldsymbol{v}):=\int_{\Omega_{\mathrm{B}}} f_{\mathrm{B}} \cdot \boldsymbol{v}_{\mathrm{B}}+\int_{\Omega_{\mathrm{D}}} f_{\mathrm{D}} \cdot \boldsymbol{v}_{\mathrm{D}},
$$

for all $\boldsymbol{u}:=\left(\boldsymbol{u}_{\mathrm{B}}, \boldsymbol{\omega}_{\mathrm{B}}, \boldsymbol{u}_{\mathrm{D}}\right), \boldsymbol{v}:=\left(\boldsymbol{v}_{\mathrm{B}}, z_{\mathrm{B}}, \boldsymbol{v}_{\mathrm{D}}\right) \in \mathbf{H}$, and for all $\mathbf{q}:=\left(q_{\mathrm{B}}, q_{\mathrm{D}}, \xi\right) \in \mathbf{Q}_{0}$.

The well-posedness of (2.2) has been established in [1] using the classical BabuškaBrezzi theory:

Theorem 1 There exists a unique $(\boldsymbol{u}, \mathbf{p}):=\left(\left(\boldsymbol{u}_{\mathrm{B}}, \boldsymbol{\omega}_{\mathrm{B}}, \boldsymbol{u}_{\mathrm{D}}\right),\left(p_{\mathrm{B}}, p_{\mathrm{D}}, \lambda\right)\right) \in \mathbf{H} \times \mathbf{Q}_{0}$ solution of the mixed formulation (2.2). Moreover, there exists $c>0$ such that

$$
\|(\boldsymbol{u}, \mathbf{p})\|_{\mathbf{H} \times \mathbf{Q}_{0}} \leq c\|\mathbf{F}\|_{\mathbf{H}^{\prime}} \leq C\left\{\left\|\boldsymbol{f}_{\mathrm{D}}\right\|_{0, \Omega_{\mathrm{D}}}+\left\|\boldsymbol{f}_{\mathrm{B}}\right\|_{0, \Omega_{\mathrm{B}}}\right\} .
$$

\subsection{Discretisation using a finite element method}

Let $\mathcal{T}_{h}^{\mathrm{B}}$ and $\mathcal{T}_{h}^{\mathrm{D}}$ be respective partitions of $\Omega_{\mathrm{B}}$ and $\Omega_{\mathrm{D}}$ by shape-regular tetrahedra $T$ of diameter $h_{T}$. We assume that these tetrahedrisations match on the interface so that $\mathcal{T}_{h}:=\mathcal{T}_{h}^{\mathrm{B}} \cup \mathcal{T}_{h}^{\mathrm{D}}$ is a regular family of triangulations of $\Omega=\Omega_{\mathrm{B}} \cup \Sigma \cup \Omega_{\mathrm{D}}$, with meshsize $h:=\max \left\{h_{T}: T \in \mathcal{T}_{h}\right\}$. We denote by $\Sigma_{h}$ the triangulation on $\Sigma$ induced by $\mathcal{T}_{h}$, which is formed by triangles $F$ of diameter $h_{F}$, and set $h_{\Sigma}:=\max \left\{h_{F}: F \in \Sigma_{h}\right\}$. Next we introduce the finite-dimensional spaces

$$
\begin{aligned}
& \mathbf{H}_{h}^{\star}:=\left\{\boldsymbol{v}_{h}^{\star} \in \mathbf{H}_{\star}\left(\operatorname{div} ; \Omega_{\star}\right):\left.\quad \boldsymbol{v}_{h}^{\star}\right|_{T} \in \mathbb{R T}_{0}(T) \quad \forall T \in \mathcal{T}_{h}^{\star}\right\}, \\
& \mathrm{Q}_{h}^{\star}:=\left\{q_{h} \in \mathrm{L}^{2}\left(\Omega_{\star}\right):\left.\quad q_{h}\right|_{T} \in P_{0}(T) \forall T \in \mathcal{T}_{h}^{\star}\right\}, \quad \mathrm{Q}_{h, 0}^{\star}:=\mathrm{Q}_{h}^{\star} \cap \mathrm{L}_{0}^{2}\left(\Omega_{\star}\right),
\end{aligned}
$$


where $\star \in\{\mathrm{B}, \mathrm{D}\}$, and for any $T \in \mathcal{T}_{h}^{\star}$ we denote by $\mathbb{R T}_{0}(T):=\mathbf{P}_{0}(T) \oplus P_{0}(T) \boldsymbol{x}$ the local Raviart-Thomas space of lowest order. In addition, we set

$$
\mathbf{H}_{0, h}^{\mathrm{B}}:=\left\{z_{h}^{\mathrm{B}} \in \mathbf{H}_{0}\left(\mathbf{c u r l} ; \Omega_{\mathrm{B}}\right):\left.\quad z_{h}^{\mathrm{B}}\right|_{T} \in \mathbb{N D}_{1}(T) \quad \forall T \in \mathcal{T}_{h}^{\mathrm{B}}\right\},
$$

where for any $T \in \mathcal{T}_{h}^{\mathrm{B}}, \mathbb{N D}_{1}(T):=\mathbf{P}_{0}(T) \oplus \mathbf{P}_{0}(T) \times \boldsymbol{x}$ is the local edge space of Nédélec type

$$
\mathbb{N D}_{1}(T):=\left\{w: T \rightarrow \mathbb{C}^{3}: \quad w(\boldsymbol{x})=\boldsymbol{a}+\boldsymbol{b} \times \boldsymbol{x} \quad \forall \boldsymbol{x} \in T, \boldsymbol{a}, \boldsymbol{b} \in \mathbb{C}^{3}\right\} .
$$

The approximation of the interface unknown will occur on an independent triangulation $\widetilde{\Sigma}_{h}$ of $\Sigma$, by elements $\widetilde{F}$ of maximum diameter $h_{\widetilde{\Sigma}}:=\max \left\{h_{\widetilde{F}}: \widetilde{F} \in \widetilde{\Sigma}_{h}\right\}$, where we define the space

$$
\mathrm{Q}_{h}^{\Sigma}:=\left\{\lambda_{h} \in \mathcal{C}(\Sigma):\left.\quad \lambda_{h}\right|_{\widetilde{F}} \in P_{1}(\widetilde{F}) \quad \forall \widetilde{F} \in \widetilde{\Sigma}_{h}\right\}
$$

In this way the Galerkin scheme associated to (2.2) reads: Find $\boldsymbol{u}_{h}:=\left(\boldsymbol{u}_{h}^{\mathrm{B}}, \boldsymbol{\omega}_{h}^{\mathrm{B}}, \boldsymbol{u}_{h}^{\mathrm{D}}\right) \in$ $\mathbf{H}_{h}$ and $\mathbf{p}_{h}:=\left(p_{h}^{\mathrm{B}}, p_{h}^{\mathrm{D}}, \lambda_{h}\right) \in \mathbf{Q}_{0, h}$ such that

$$
\begin{array}{ll}
\mathbf{a}\left(\boldsymbol{u}_{h}, \boldsymbol{v}_{h}\right)+\mathbf{b}\left(\boldsymbol{v}_{h}, \mathbf{p}_{h}\right)=\mathbf{F}\left(\boldsymbol{v}_{h}\right) & \forall \boldsymbol{v}_{h}:=\left(\boldsymbol{v}_{h}^{\mathrm{B}}, \boldsymbol{z}_{h}^{\mathrm{B}}, \boldsymbol{v}_{h}^{\mathrm{D}}\right) \in \mathbf{H}_{h}, \\
\mathbf{b}\left(\boldsymbol{u}_{h}, \mathbf{q}_{h}\right)=\mathbf{0} & \forall \mathbf{q}_{h}:=\left(q_{h}^{\mathrm{B}}, q_{h}^{\mathrm{D}}, \xi_{h}\right) \in \mathbf{Q}_{0, h},
\end{array}
$$

where $\mathbf{H}_{h}:=\mathbf{H}_{h}^{\mathrm{B}} \times \mathbf{H}_{0, h}^{\mathrm{B}} \times \mathbf{H}_{h}^{\mathrm{D}}$ and $\mathbf{Q}_{0, h}:=\mathrm{Q}_{h, 0}^{\mathrm{B}} \times \mathrm{Q}_{h}^{\mathrm{D}} \times \mathrm{Q}_{h}^{\Sigma}$. We point out that the solvability of (2.9) requires the mesh condition $h_{\Sigma} \leq C_{0} h_{\widetilde{\Sigma}}$, where $C_{0}$ is a positive constant. Details are to be found in $[1, \S 4.2 .3-4.2 .4]$.

\section{A residual-based a posteriori error estimator}

In this section we derive a reliable and efficient a posteriori error estimator for the Galerkin scheme (2.9). Most of the present proofs make extensive use of estimates available in $[1,3,5,6,8,15,21,23,24,27]$.

\subsection{Preliminaries}

Given a tetrahedron $T \in \mathcal{T}_{h}$, we let $\mathcal{E}(T)$ and $\mathcal{F}(T)$ be the sets of its edges and faces, respectively. In addition, we denote by $\mathcal{E}_{h}$ and $\mathcal{F}_{h}$ be the sets of all edges and faces of $\mathcal{T}_{h}$, respectively, so that $\mathcal{F}_{h}$ is subdivided as follows:

$$
\mathcal{F}_{h}=\mathcal{F}_{h}\left(\Gamma_{\mathrm{B}}\right) \cup \mathcal{F}_{h}\left(\Omega_{\mathrm{B}}\right) \cup \mathcal{F}_{h}(\Sigma) \cup \mathcal{F}_{h}\left(\Omega_{\mathrm{D}}\right) \cup \mathcal{F}_{h}\left(\Gamma_{\mathrm{D}}\right),
$$

where $\mathcal{F}_{h}\left(\Gamma_{\star}\right):=\left\{F \in \mathcal{F}_{h}: F \subseteq \Gamma_{\star}\right\}, \mathcal{F}_{h}\left(\Omega_{\star}\right):=\left\{F \in \mathcal{F}_{h}: F \subseteq \Omega_{\star}\right\}$, for each $\star \in\{\mathrm{B}, \mathrm{D}\}$, and $\mathcal{F}_{h}(\Sigma):=\left\{F \in \overline{\mathcal{F}}_{h}: F \subseteq \Sigma\right\}$. In turn, for each $T \in \mathcal{T}_{h}$ we denote

$$
\mathcal{F}_{h, T}\left(\Omega_{\star}\right):=\left\{F \in \partial T: F \in \mathcal{F}_{h}\left(\Omega_{\star}\right)\right\}, \quad \mathcal{F}_{h, T}\left(\Gamma_{\star}\right):=\left\{F \in \partial T: F \in \mathcal{F}_{h}\left(\Gamma_{\star}\right)\right\}
$$


and $\mathcal{F}_{h, T}(\Sigma):=\left\{F \in \partial T: F \in \mathcal{F}_{h}(\Sigma)\right\}$. Also, for each face $F \in \mathcal{F}_{h}\left(\Omega_{\star}\right)$ we fix a unit normal $\boldsymbol{n}_{F}$ to $F$, so that given $\boldsymbol{v} \in \mathbf{L}^{2}\left(\Omega_{\star}\right)$ such that $\left.\boldsymbol{v}\right|_{T} \in \mathbf{C}(T)$ on each $T \in \mathcal{T}_{h}^{\star}$, and given $F \in \mathcal{F}_{h}\left(\Omega_{\star}\right)$, we let $\llbracket \boldsymbol{v} \times \boldsymbol{n}_{F} \rrbracket$ be the corresponding jump of the tangential traces across $F$, that is $\llbracket \boldsymbol{v} \times \boldsymbol{n}_{F} \rrbracket:=\left.\left(\left.\boldsymbol{v}\right|_{T}-\left.\boldsymbol{v}\right|_{T^{\prime}}\right)\right|_{F} \times \boldsymbol{n}_{F}$, where $T$ and $T^{\prime}$ are the elements of $\mathcal{T}_{h}^{\star}$ having $F$ as a common face. In addition, for each edge $E$ of a tetrahedron $T \in \mathcal{T}_{h}^{\star}$, we fix a unit tangential vector $\boldsymbol{t}_{E}$ along $E$. When no confusion arises, we simple write $\boldsymbol{n}$ instead of $\boldsymbol{n}_{F}$, and $\boldsymbol{t}$ instead $\boldsymbol{t}_{E}$.

We now recall from [13] the tangential curl operator $\operatorname{curl}_{s}: \mathrm{H}^{1 / 2}(\Sigma) \rightarrow$ $\mathcal{L}\left(\mathrm{H}^{-1 / 2}(\Sigma)\right)$, with $\mathcal{L}\left(\mathrm{H}^{-1 / 2}(\Sigma)\right)$ denoting the tangential vector fields of order $-1 / 2$, which is defined by $\operatorname{curl}_{s}(\chi):=\nabla \chi \times \boldsymbol{n}$, for any sufficiently smooth function $\chi$. This is a linear and continuous map (see [13, Propositions 3.4 and 3.6]) which will be required in the sequel. We will also make use of the Raviart-Thomas interpolator of lowest order (see [22]) $\Pi_{h}^{\star}: \mathbf{H}^{1}\left(\Omega_{\star}\right) \rightarrow \mathbf{H}_{h}^{\star}, \star \in\{\mathrm{B}, \mathrm{D}\}$, which according to its characterisation given by the identity

$$
\int_{F} \Pi_{h}^{\star} \boldsymbol{v} \cdot \boldsymbol{n}=\int_{F} \boldsymbol{v} \cdot \boldsymbol{n} \quad \forall \text { face } F \text { of } \mathcal{T}_{h}^{\star},
$$

verifies that

$$
\operatorname{div}\left(\Pi_{h}^{\star} v\right)=\mathcal{P}_{h}^{\star}(\operatorname{div} v),
$$

where $\mathcal{P}_{h}^{\star}$ is the $\mathrm{L}^{2}\left(\Omega_{\star}\right)$-orthogonal projector onto $\mathrm{P}_{0}\left(\Omega_{\star}\right)$. In addition, we recall the Clément operator onto the space of the continuous piecewise linear functions $\mathrm{I}_{h}^{\star}: \mathrm{H}^{1}\left(\Omega_{\star}\right) \rightarrow \mathrm{X}_{h}^{\star}$ (cf. [20]), where

$$
\mathrm{X}_{h}^{\star}:=\left\{v \in \mathcal{C}\left(\bar{\Omega}_{\star}\right):\left.\quad v\right|_{T} \in \mathrm{P}_{1}(T) \quad \forall T \in \mathcal{T}_{h}^{\star}\right\},
$$

and let $\mathbf{I}_{h}^{\star}: \mathbf{H}^{1}\left(\Omega_{\star}\right) \rightarrow \mathbf{X}_{h}^{\star}$ be its vectorial counterpart defined component-wise. These maps satisfy the following properties (see [12,20,22], respectively)

Lemma 1 There exist $c_{1}, c_{2}>0$, independent of $h$, such that for all $\boldsymbol{v} \in \mathbf{H}^{1}\left(\Omega_{\star}\right)$ there hold

$$
\begin{gathered}
\left\|\boldsymbol{v}-\Pi_{h}^{\star} \boldsymbol{v}\right\|_{0, T} \leq c_{1} h_{T}\|\boldsymbol{v}\|_{1, T} \quad \forall T \in \mathcal{T}_{h}^{\star}, \\
\left\|\boldsymbol{v} \cdot \boldsymbol{n}-\Pi_{h}^{\star} \boldsymbol{v} \cdot \boldsymbol{n}\right\|_{0, F} \leq c_{2} h_{F}^{1 / 2}\|\boldsymbol{v}\|_{1, T_{F}} \quad \forall F \text { of } \mathcal{T}_{h}^{\star},
\end{gathered}
$$

where $T_{F}$ is a tetrahedron of $\mathcal{T}_{h}^{\star}$ containing a face $F$ on its boundary.

Lemma 2 There exist constants $c_{3}, c_{4}>0$, independent of $h$, such that for all $v \in \mathrm{H}^{1}\left(\Omega_{\star}\right)$ there hold

$$
\begin{aligned}
& \left\|v-\mathrm{I}_{h}^{\star}(v)\right\|_{0, T} \leq c_{3} h_{T}\|v\|_{1, \Delta_{\star}(T)} \quad \forall T \in \mathcal{T}_{h}^{\star}, \\
& \left\|v-\mathrm{I}_{h}^{\star}(v)\right\|_{0, F} \leq c_{4} h_{F}^{1 / 2}\|v\|_{1, \Delta_{\star}(F)} \quad \forall F \in \mathcal{F}_{h}\left(\Omega_{\star}\right),
\end{aligned}
$$


where

$$
\begin{array}{ll}
\Delta_{\star}(T):=\cup\left\{T^{\prime} \in \mathcal{T}_{h}^{\star}:\right. & \left.T^{\prime} \cap T \neq \emptyset\right\} \text { and } \\
\Delta_{\star}(F):=\cup\left\{T^{\prime} \in \mathcal{T}_{h}^{\star}:\right. & \left.T^{\prime} \cap F \neq \emptyset\right\} .
\end{array}
$$

Furthermore, following [21] we define the Clément-type Nédélec interpolator $\mathcal{N}_{h}$ : $\mathbf{L}^{2}\left(\Omega_{\mathrm{B}}\right) \rightarrow \mathbf{H}_{h, 0}^{\mathrm{B}}$ by:

$$
\mathcal{N}_{h}(\boldsymbol{\psi}):=\sum_{E \in \mathcal{E}_{h}\left(\Omega_{\mathrm{B}}\right)}\left(\frac{1}{\left|\Delta_{\mathrm{B}}(E)\right|} \int_{\Delta_{\mathrm{B}}(E)} \boldsymbol{\psi} \cdot \mathbf{t}_{E}\right)|E| \lambda_{E},
$$

where $\mathcal{E}_{h}\left(\Omega_{\mathrm{B}}\right)$ is the set of interior edges of $\mathcal{T}_{h}^{\mathrm{B}}, \Delta_{\mathrm{B}}(E):=\cup\left\{T^{\prime} \in \mathcal{T}_{h}^{\mathrm{B}}: T^{\prime} \cap E \neq \emptyset\right\}$, and $\lambda_{E}$ is the standard basis function for the lowest order Nédelec element, which satisfies

$$
\int_{E^{\prime}} \lambda_{E} \cdot \mathbf{t}_{E^{\prime}}=\delta_{E, E^{\prime}} \quad \forall E^{\prime} \in \mathcal{E}(T)
$$

where $\delta_{E, E^{\prime}}$ is the Kronecker delta. The approximation properties of $\mathcal{N}_{h}$ are summarised in the following Lemma (see [21, §4.3, Theorem 4.2, and §6] and also [8, Proposition 2]).

Lemma 3 There exist $c_{5}, c_{6}>0$, independent of $h$, such that for all $\boldsymbol{\psi} \in$ $\mathbf{H}_{0}\left(\mathbf{c u r l}, \Omega_{\mathrm{B}}\right) \cap \mathbf{H}^{1}\left(\Omega_{\mathrm{B}}\right)$,

$$
\begin{aligned}
& \left\|\boldsymbol{\psi}-\mathcal{N}_{h}(\boldsymbol{\psi})\right\|_{0, T} \leq c_{5} h_{T}\|\boldsymbol{\psi}\|_{1, \Delta_{\mathrm{B}}(T)} \quad \forall T \in \mathcal{T}_{h}^{\mathrm{B}}, \\
& \left\|\boldsymbol{\psi}-\mathcal{N}_{h}(\boldsymbol{\psi})\right\|_{0, F} \leq c_{6} h_{F}^{1 / 2}\|\boldsymbol{\psi}\|_{1, \Delta_{\mathrm{B}}(F)} \quad \forall F \in \mathcal{F}_{h}\left(\Omega_{\mathrm{B}}\right)
\end{aligned}
$$

We will also require stable Helmholtz decompositions for $\mathbf{H}_{\star}\left(\operatorname{div} ; \Omega_{\star}\right)$ with $* \epsilon$ $\{\mathrm{B}, \mathrm{D}\}$. A technical assumption is that $\Gamma_{\star}$ lies on the "convex part" of $\Omega_{\star}$, signifying that there exists a convex domain containing $\Omega_{\star}$, whose boundary contains $\Gamma_{\star}$. More precisely, introducing the space

$$
\mathbf{H}_{\Gamma_{\star}}^{1}\left(\Omega_{\star}\right):=\left\{\boldsymbol{\beta} \in \mathbf{H}^{1}\left(\Omega_{\star}\right):\left.\quad \boldsymbol{\beta}\right|_{\Gamma_{\star}}=\mathbf{0}\right\},
$$

we have the following result shown in [23, Theorem 3.2].

Lemma 4 Assume that there exists a convex domain $\Xi_{\star}$ such that $\Omega_{\star} \subseteq \Xi_{\star}$ and $\Gamma_{\star} \subseteq \partial \Xi_{\star}$. Then, given $\boldsymbol{v}_{\star} \in \mathbf{H}_{\star}\left(\right.$ div; $\left.\Omega_{\star}\right)$ there exist $w \in \mathrm{H}^{2}\left(\Omega_{\star}\right)$ and $\boldsymbol{\beta} \in \mathbf{H}_{\Gamma_{\star}}^{1}\left(\Omega_{\star}\right)$ such that

$$
\boldsymbol{v}_{\star}=\nabla w+\operatorname{curl} \boldsymbol{\beta} \text { in } \Omega_{\star} \text { and }\|w\|_{2, \Omega_{\star}}+\|\boldsymbol{\beta}\|_{1, \Omega_{\star}} \leq C_{\star}\left\|\boldsymbol{v}_{\star}\right\|_{\operatorname{div}, \Omega_{\star}}
$$

where $C_{\star}$ is a positive constant independent of all the foregoing variables. 
In turn, a decomposition for $\mathbf{H}_{0}\left(\mathbf{c u r l} ; \Omega_{\mathrm{B}}\right)$ is given as follows.

Lemma 5 Given $z_{\mathrm{B}} \in \mathbf{H}_{0}\left(\mathbf{c u r l} ; \Omega_{\mathrm{B}}\right)$ there exist $\boldsymbol{\varphi} \in \mathbf{H}_{0}^{1}\left(\Omega_{\mathrm{B}}\right), \chi \in \mathrm{H}_{0}^{1}\left(\Omega_{\mathrm{B}}\right)$, and $C>0$ such that

$$
z_{\mathrm{B}}=\boldsymbol{\varphi}+\nabla \chi \text { in } \Omega_{\mathrm{B}},
$$

and

$$
\|\varphi\|_{1, \Omega_{\mathrm{B}}}+\|\chi\|_{1, \Omega_{\mathrm{B}}} \leq C\left\|z_{\mathrm{B}}\right\|_{\text {curl }, \Omega_{\mathrm{B}}} .
$$

Proof See [31, Lemma 2.2 and §5]

We end this section with an estimate (in terms of local quantities) for the $\mathrm{H}^{-1 / 2}(\Sigma)$ norm of functions in a particular subspace of $\mathrm{H}^{-1 / 2}(\Sigma) \cap \mathrm{L}^{2}(\Sigma)$. According to the definition of $\mathrm{Q}_{h}^{\Sigma}$ (cf. 2.8), we introduce the following orthogonal-type space

$$
\mathrm{Q}_{h}^{\Sigma, \perp}:=\left\{\varphi \in \mathrm{H}^{-1 / 2}(\Sigma) \cap \mathrm{L}^{2}(\Sigma): \quad\left\langle\varphi, \lambda_{h}\right\rangle_{\Sigma}=0 \quad \forall \lambda_{h} \in \mathrm{Q}_{h}^{\Sigma}\right\} .
$$

Lemma 6 Assume that for each $F \in \Sigma_{h}$ there exists $\widetilde{F} \in \widetilde{\Sigma}_{h}$ such that $F \subseteq \widetilde{F}$ and $h_{\Sigma} \leq C_{1} h_{\widetilde{\Sigma}}$, with a constant $C_{1}>0$ independent of $h_{\Sigma}$ and $h_{\tilde{\Sigma}}$. Then, there exists $C>0$ independent of the aforementioned meshsizes, such that

$$
\|\varphi\|_{-1 / 2, \Sigma}^{2} \leq C \sum_{F \in \Sigma_{h}} h_{F}\|\varphi\|_{0, F}^{2} \quad \forall \varphi \in \mathrm{Q}_{h}^{\Sigma, \perp}(\Sigma) .
$$

Proof See [16, Lemma 3.4].

\subsection{Defining the proposed estimator}

Given $\left(\boldsymbol{u}_{h}, \mathbf{p}_{h}\right):=\left(\left(\boldsymbol{u}_{h}^{\mathrm{B}}, \boldsymbol{\omega}_{h}^{\mathrm{B}}, \boldsymbol{u}_{h}^{\mathrm{D}}\right),\left(p_{h}^{\mathrm{B}}, p_{h}^{\mathrm{D}}, \lambda_{h}\right)\right) \in \mathbf{H}_{h} \times \mathbf{Q}_{h, 0}$ the unique solution of (2.9), we define for each $T \in \mathcal{T}_{h}^{\mathrm{B}}$, the local a posteriori error indicator $\Theta_{\mathrm{B}, T}$ as follows:

$$
\begin{aligned}
\Theta_{\mathrm{B}, T}^{2}:= & h_{T}^{2}\left\|\boldsymbol{f}_{\mathrm{B}}-\kappa_{\mathrm{B}}^{-1} \boldsymbol{u}_{h}^{\mathrm{B}}-v \operatorname{curl} \boldsymbol{\omega}_{h}^{\mathrm{B}}\right\|_{0, T}^{2}+h_{T}^{2}\left\|\operatorname{curl} \boldsymbol{u}_{h}^{\mathrm{B}}-\boldsymbol{\omega}_{h}^{\mathrm{B}}\right\|_{0, T}^{2} \\
& +\left\|\operatorname{div} \boldsymbol{u}_{h}^{\mathrm{B}}\right\|_{0, T}^{2}+h_{T}^{2}\left\|\operatorname{curl}\left\{\boldsymbol{f}_{\mathrm{B}}-\kappa_{\mathrm{B}}^{-1} \boldsymbol{u}_{h}^{\mathrm{B}}-v \operatorname{curl} \boldsymbol{\omega}_{h}^{\mathrm{B}}\right\}\right\|_{0, T}^{2} \\
& +\sum_{F \in \mathcal{F}_{h, T}\left(\Omega_{\mathrm{B}}\right)} h_{F}\left\{\left\|\llbracket \boldsymbol{u}_{h}^{\mathrm{B}} \times \boldsymbol{n} \rrbracket\right\|_{0, F}^{2}+\left\|\llbracket \boldsymbol{\omega}_{h}^{\mathrm{B}} \cdot \boldsymbol{n} \rrbracket\right\|_{0, F}^{2}\right\} \\
& +\sum_{F \in \mathcal{F}_{h, T}\left(\Omega_{\mathrm{B}}\right)} h_{F}\left\|\llbracket\left(\boldsymbol{f}_{\mathrm{B}}-\kappa_{\mathrm{B}}^{-1} \boldsymbol{u}_{h}^{\mathrm{B}}-v \operatorname{curl} \boldsymbol{\omega}_{h}^{\mathrm{B}}\right) \times \boldsymbol{n} \rrbracket\right\|_{0, F}^{2} \\
& +\sum_{F \in \mathcal{F}_{h, T}\left(\Gamma_{\mathrm{B}}\right)} h_{F}\left\|\left(\boldsymbol{f}_{\mathrm{B}}-\kappa_{\mathrm{B}}^{-1} \boldsymbol{u}_{h}^{\mathrm{B}}-v \operatorname{curl} \boldsymbol{\omega}_{h}^{\mathrm{B}}\right) \times \boldsymbol{n}\right\|_{0, F}^{2} \\
& +\sum_{F \in \mathcal{F}_{h, T}(\Sigma)} h_{F}\left\{\left\|\left(\boldsymbol{f}_{\mathrm{B}}-\kappa_{\mathrm{B}}^{-1} \boldsymbol{u}_{h}^{\mathrm{B}}-v \operatorname{curl} \boldsymbol{\omega}_{h}^{\mathrm{B}}\right) \times \boldsymbol{n}-\operatorname{curl}_{s}\left(\lambda_{h}\right)\right\|_{0, F}^{2}\right. \\
& \left.+\left\|p_{h}^{\mathrm{B}}-\lambda_{h}\right\|_{0, F}^{2}\right\}
\end{aligned}
$$


and for each $T \in \mathcal{T}_{h}^{\mathrm{D}}$, we define the local a posteriori error indicator $\Theta_{\mathrm{D}, T}$ as

$$
\begin{aligned}
\Theta_{\mathrm{D}, T}^{2}:= & h_{T}^{2}\left\|\boldsymbol{f}_{\mathrm{D}}-\kappa_{\mathrm{D}}^{-1} \boldsymbol{u}_{h}^{\mathrm{D}}\right\|_{0, T}^{2}+h_{T}^{2}\left\|\operatorname{curl}\left\{\boldsymbol{f}_{\mathrm{D}}-\kappa_{\mathrm{D}}^{-1} \boldsymbol{u}_{h}^{\mathrm{D}}\right\}\right\|_{0, T}^{2}+\left\|\operatorname{div} \boldsymbol{u}_{h}^{\mathrm{D}}\right\|_{0, T}^{2} \\
& +\sum_{F \in \mathcal{F}_{h, T}\left(\Omega_{\mathrm{D}}\right)} h_{F}\left\|\llbracket\left(\boldsymbol{f}_{\mathrm{D}}-\kappa_{\mathrm{D}}^{-1} \boldsymbol{u}_{h}^{\mathrm{D}}\right) \times \boldsymbol{n} \rrbracket\right\|_{0, F}^{2} \\
& +\sum_{F \in \mathcal{F}_{h, T}\left(\Gamma_{\mathrm{D}}\right)} h_{F}\left\|\left(\boldsymbol{f}_{\mathrm{D}}-\kappa_{\mathrm{D}}^{-1} \boldsymbol{u}_{h}^{\mathrm{D}}\right) \times \boldsymbol{n}\right\|_{0, F}^{2} \\
& +\sum_{F \in \mathcal{F}_{h, T}(\Sigma)} h_{F}\left\{\left\|\left(\boldsymbol{f}_{\mathrm{D}}-\kappa_{\mathrm{D}}^{-1} \boldsymbol{u}_{h}^{\mathrm{D}}\right) \times \boldsymbol{n}-\operatorname{curl}_{s}\left(\lambda_{h}\right)\right\|_{0, F}^{2}\right. \\
& \left.+\left\|p_{h}^{\mathrm{D}}-\lambda_{h}\right\|_{0, F}^{2}+\left\|\boldsymbol{u}_{h}^{\mathrm{B}} \cdot \boldsymbol{n}-\boldsymbol{u}_{h}^{\mathrm{D}} \cdot \boldsymbol{n}\right\|_{0, F}^{2}\right\} .
\end{aligned}
$$

It is not difficult to see that each term defining $\Theta_{\mathrm{B}, T}^{2}$ and $\Theta_{\mathrm{D}, T}^{2}$ is residual. Hence a global residual error estimator for (2.9) can be defined as

$$
\Theta:=\left\{\sum_{T \in \mathcal{T}_{h}^{\mathrm{B}}} \Theta_{\mathrm{B}, T}^{2}+\sum_{T \in \mathcal{T}_{h}^{\mathrm{D}}} \Theta_{\mathrm{D}, T}^{2}\right\}^{\frac{1}{2}} .
$$

The remainder of this section advocates to establish the existence of positive constants $C_{\text {eff }}$ and $C_{\text {rel }}$, independent of the meshsizes and the continuous and discrete solutions, such that

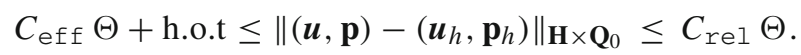

where h.o.t stands, eventually, for one or several terms of higher order. The upper and lower bounds in (3.8), are derived below in Sects. 3.3 and 3.4, respectively.

\subsection{Reliability}

\subsubsection{Preliminary estimates}

We begin by recalling that the first inequality in the continuous dependence result (2.3) is equivalent to the global inf-sup condition

$$
\frac{1}{c}\|(\boldsymbol{w}, \mathbf{r})\|_{\mathbf{H} \times \mathbf{Q}_{0}} \leq \sup _{\substack{(\boldsymbol{v}, \mathbf{q}) \in \mathbf{H} \times \mathbf{Q}_{0} \\(\boldsymbol{v}, \mathbf{q}) \neq \mathbf{0}}} \frac{\mathbf{a}(\boldsymbol{w}, \boldsymbol{v})+\mathbf{b}(\boldsymbol{v}, \mathbf{r})+\mathbf{b}(\boldsymbol{w}, \mathbf{q})}{\|(\boldsymbol{v}, \mathbf{q})\|_{\mathbf{H} \times \mathbf{Q}_{0}}}
$$

for all $(\boldsymbol{w}, \mathbf{r}) \in \mathbf{H} \times \mathbf{Q}_{0}$. This allows to establish a first estimate for the total error as follows.

Theorem 2 Let $(\boldsymbol{u}, \mathbf{p}) \in \mathbf{H} \times \mathbf{Q}_{0}$ and $\left(\boldsymbol{u}_{h}, \mathbf{p}_{h}\right) \in \mathbf{H}_{h} \times \mathbf{Q}_{0, h}$ be the unique solutions of (2.2) and (2.9), respectively. Then, there exists a constant $C>0$, independent of $h$, such that 


$$
\begin{aligned}
\left\|(\boldsymbol{u}, \mathbf{p})-\left(\boldsymbol{u}_{h}, \mathbf{p}_{h}\right)\right\|_{\mathbf{H} \times \mathbf{Q}_{0} \leq} & C\left\{\|\mathbf{E}\|_{\mathbf{H}^{\prime}}+\left\|\boldsymbol{u}_{h}^{\mathrm{B}} \cdot \boldsymbol{n}-\boldsymbol{u}_{h}^{\mathrm{D}} \cdot \boldsymbol{n}\right\|_{-1 / 2, \Sigma}\right. \\
& \left.+\left\|\operatorname{div} \boldsymbol{u}_{h}^{\mathrm{B}}\right\|_{0, \Omega_{\mathrm{B}}}+\left\|\operatorname{div} \boldsymbol{u}_{h}^{\mathrm{D}}\right\|_{0, \Omega_{\mathrm{D}}}\right\},
\end{aligned}
$$

where $\mathbf{E} \in \mathbf{H}^{\prime}$ is defined by

$$
\mathbf{E}(\boldsymbol{v}):=\mathbf{F}(\boldsymbol{v})-\mathbf{a}\left(\boldsymbol{u}_{h}, \boldsymbol{v}\right)-\mathbf{b}\left(\boldsymbol{v}, \mathbf{p}_{h}\right) \quad \forall \boldsymbol{v} \in \mathbf{H},
$$

and satisfies

$$
\mathbf{E}\left(\boldsymbol{v}_{h}\right)=0 \quad \forall \boldsymbol{v}_{h} \in \mathbf{H}_{h} .
$$

Proof Applying (3.9) to the error $(\boldsymbol{w}, \mathbf{r}):=(\boldsymbol{u}, \mathbf{p})-\left(\boldsymbol{u}_{h}, \mathbf{p}_{h}\right)$ and using (3.10) we arrive at

$$
\frac{1}{c}\left\|(\boldsymbol{u}, \mathbf{p})-\left(\boldsymbol{u}_{h}, \mathbf{p}_{h}\right)\right\|_{\mathbf{H} \times \mathbf{Q}_{0}} \leq \sup _{\substack{(\boldsymbol{v}, \mathbf{q}) \in \mathbf{H} \times \mathbf{Q}_{0} \\(\boldsymbol{v}, \mathbf{q}) \neq \mathbf{0}}} \frac{\mathbf{E}(\boldsymbol{v})+\mathbf{b}\left(\boldsymbol{u}-\boldsymbol{u}_{h}, \mathbf{q}\right)}{\|(\boldsymbol{v}, \mathbf{q})\|_{\mathbf{H} \times \mathbf{Q}_{0}}} .
$$

Then, noting that obviously

$$
\frac{|\mathbf{E}(\boldsymbol{v})|}{\|(\boldsymbol{v}, \mathbf{q})\|_{\mathbf{H} \times \mathbf{Q}_{0}}} \leq \frac{|\mathbf{E}(\boldsymbol{v})|}{\|\boldsymbol{v}\|_{\mathbf{H}}} \quad \text { and } \quad \frac{\left|\mathbf{b}\left(\boldsymbol{u}-\boldsymbol{u}_{h}, \mathbf{q}\right)\right|}{\|(\boldsymbol{v}, \mathbf{q})\|_{\mathbf{H} \times \mathbf{Q}_{0}}} \leq \frac{\left|\mathbf{b}\left(\boldsymbol{u}-\boldsymbol{u}_{h}, \mathbf{q}\right)\right|}{\|\mathbf{q}\|_{\mathbf{Q}_{0}}}
$$

and applying the supremum in (3.12), we find that

$$
\frac{1}{c}\left\|(\boldsymbol{u}, \mathbf{p})-\left(\boldsymbol{u}_{h}, \mathbf{p}_{h}\right)\right\|_{\mathbf{H} \times \mathbf{Q}_{0}} \leq\|\mathbf{E}\|_{\mathbf{H}^{\prime}}+\left\|\mathbf{b}\left(\boldsymbol{u}-\boldsymbol{u}_{h}, \cdot\right)\right\|_{\mathbf{Q}_{0}^{\prime}} \cdot
$$

Next, employing the second equation of (2.2) and the definition of $\mathbf{b}$, we deduce that

$$
\mathbf{b}\left(\boldsymbol{u}-\boldsymbol{u}_{h}, \mathbf{q}\right)=-\int_{\Omega_{\mathrm{B}}} q_{\mathrm{B}} \operatorname{div} \boldsymbol{u}_{h}^{\mathrm{B}}-\int_{\Omega_{\mathrm{D}}} q_{\mathrm{D}} \operatorname{div} \boldsymbol{u}_{h}^{\mathrm{D}}+\left\langle\boldsymbol{u}_{h}^{\mathrm{B}} \cdot \boldsymbol{n}-\boldsymbol{u}_{h}^{\mathrm{D}} \cdot \boldsymbol{n}, \xi\right\rangle_{\Sigma},
$$

which yields

$$
\left\|\mathbf{b}\left(\boldsymbol{u}-\boldsymbol{u}_{h}, \cdot\right)\right\|_{\mathbf{Q}_{0}^{\prime}} \leq\left\|\boldsymbol{u}_{h}^{\mathrm{B}} \cdot \boldsymbol{n}-\boldsymbol{u}_{h}^{\mathrm{D}} \cdot \boldsymbol{n}\right\|_{-1 / 2, \Sigma}+\left\|\operatorname{div} \boldsymbol{u}_{h}^{\mathrm{B}}\right\|_{0, \Omega_{\mathrm{B}}}+\left\|\operatorname{div} \boldsymbol{u}_{h}^{\mathrm{D}}\right\|_{0, \Omega_{\mathrm{D}}} \cdot
$$

Finally, from (3.10) and the first equation of (2.9), we obtain (3.11), and the proof concludes.

The next step consists in deriving suitable upper bounds for the residual term $\left\|\boldsymbol{u}_{h}^{\mathrm{B}} \cdot \boldsymbol{n}-\boldsymbol{u}_{h}^{\mathrm{D}} \cdot \boldsymbol{n}\right\|_{-1 / 2, \Sigma}$ and for $\|\mathbf{E}\|_{\mathbf{H}^{\prime}}$. We begin with the following result.

Lemma 7 There exists $C_{4}>0$, independent of the meshsizes, such that

$$
\left\|\boldsymbol{u}_{h}^{\mathrm{B}} \cdot \boldsymbol{n}-\boldsymbol{u}_{h}^{\mathrm{D}} \cdot \boldsymbol{n}\right\|_{-1 / 2, \Sigma} \leq C_{4}\left\{\sum_{F \in \mathcal{F}_{h}(\Sigma)} h_{F}\left\|\boldsymbol{u}_{h}^{\mathrm{B}} \cdot \boldsymbol{n}-\boldsymbol{u}_{h}^{\mathrm{D}} \cdot \boldsymbol{n}\right\|_{0, F}^{2}\right\}^{1 / 2} .
$$


Proof Taking $\xi_{h} \in \mathrm{Q}_{h}^{\Sigma}$ and then $\mathbf{p}_{h}=\left(0,0, \xi_{h}\right) \in \mathbf{Q}_{h, 0}$ in the second equation of (2.9), we find that

$$
\left\langle\boldsymbol{u}_{h}^{\mathrm{B}} \cdot \boldsymbol{n}-\boldsymbol{u}_{h}^{\mathrm{D}} \cdot \boldsymbol{n}, \xi_{h}\right\rangle_{\Sigma}=0 \quad \forall \xi_{h} \in \mathrm{Q}_{h}^{\Sigma},
$$

which says that each component of $\boldsymbol{u}_{h}^{\mathrm{B}} \cdot \boldsymbol{n}-\boldsymbol{u}_{h}^{\mathrm{D}} \cdot \boldsymbol{n}$ belongs to $\mathrm{Q}_{h}^{\Sigma, \perp}$ (cf. 3.3). In this way, (3.13) follows from a direct component-wise application of (3.4) (cf. Lemma 6).

We now aim to estimate $\|\mathbf{E}\|_{\mathbf{H}^{\prime}}$. To this end, we first rewrite the functional as follows

$$
\mathbf{E}(\boldsymbol{v})=\mathbf{E}_{1}\left(\boldsymbol{v}_{\mathrm{B}}\right)+\mathbf{E}_{2}\left(z_{\mathrm{B}}\right)+\mathbf{E}_{3}\left(\boldsymbol{v}_{\mathrm{D}}\right),
$$

where $\mathbf{E}_{1} \in \mathbf{H}_{\mathrm{B}}\left(\operatorname{div} ; \Omega_{\mathrm{B}}\right)^{\prime}, \mathbf{E}_{2} \in \mathbf{H}_{0}\left(\mathbf{c u r l} ; \Omega_{\mathrm{B}}\right)^{\prime}$ and $\mathbf{E}_{3} \in \mathbf{H}_{\mathrm{D}}\left(\operatorname{div} ; \Omega_{\mathrm{D}}\right)^{\prime}$ are defined by

$$
\begin{aligned}
\mathbf{E}_{1}\left(\boldsymbol{v}_{\mathrm{B}}\right) & :=\int_{\Omega_{\mathrm{B}}}\left(\boldsymbol{f}_{\mathrm{B}}-\kappa_{\mathrm{B}}^{-1} \boldsymbol{u}_{h}^{\mathrm{B}}-v \operatorname{curl} \boldsymbol{\omega}_{h}^{\mathrm{B}}\right) \cdot \boldsymbol{v}_{\mathrm{B}}+\int_{\Omega_{\mathrm{B}}} p_{h}^{\mathrm{B}} \operatorname{div} \boldsymbol{v}_{\mathrm{B}}-\left\langle\boldsymbol{v}_{\mathrm{B}} \cdot \boldsymbol{n}, \lambda_{h}\right\rangle_{\Sigma}, \\
\mathbf{E}_{2}\left(z_{\mathrm{B}}\right) & :=v \int_{\Omega_{\mathrm{B}}} \boldsymbol{u}_{h}^{\mathrm{B}} \cdot \operatorname{curl} z_{\mathrm{B}}-v \int_{\Omega_{\mathrm{B}}} \boldsymbol{\omega}_{h}^{\mathrm{B}} \cdot z_{\mathrm{B}}, \\
\mathbf{E}_{3}\left(\boldsymbol{v}_{\mathrm{D}}\right) & :=\int_{\Omega_{\mathrm{D}}}\left(f_{\mathrm{D}}-\kappa_{\mathrm{D}}^{-1} \boldsymbol{u}_{h}^{\mathrm{D}}\right) \cdot \boldsymbol{v}_{\mathrm{D}}+\int_{\Omega_{\mathrm{B}}} p_{h}^{\mathrm{D}} \operatorname{div} \boldsymbol{v}_{\mathrm{D}}+\left\langle\boldsymbol{v}_{\mathrm{D}} \cdot \boldsymbol{n}, \lambda_{h}\right\rangle_{\Sigma} .
\end{aligned}
$$

Notice, from (3.11), that $\forall \boldsymbol{v}_{h}:=\left(\boldsymbol{v}_{h}^{\mathrm{B}}, z_{h}^{\mathrm{B}}, \boldsymbol{v}_{h}^{\mathrm{D}}\right) \in \mathbf{H}_{h}$, there holds

$$
\mathbf{E}_{1}\left(\boldsymbol{v}_{h}^{\mathrm{B}}\right)+\mathbf{E}_{2}\left(z_{h}^{\mathrm{B}}\right)+\mathbf{E}_{3}\left(\boldsymbol{v}_{h}^{\mathrm{D}}\right)=0 .
$$

\subsubsection{Upper bound for $\left\|\mathbf{E}_{1}\right\|_{\mathbf{H}_{\mathrm{B}}\left(\mathrm{div} ; \Omega_{\mathrm{B}}\right)^{\prime}}$}

Given $\boldsymbol{v}_{\mathrm{B}} \in \mathbf{H}_{\mathrm{B}}\left(\operatorname{div} ; \Omega_{\mathrm{B}}\right)$, we consider its Helmholtz decomposition established in Lemma 4. More precisely, we let $w \in \mathrm{H}^{2}\left(\Omega_{\mathrm{B}}\right)$ and $\boldsymbol{\beta} \in \mathbf{H}_{\Gamma_{\mathrm{B}}}^{1}\left(\Omega_{\mathrm{B}}\right)$ be such that $\boldsymbol{v}_{\mathrm{B}}=\nabla w+\operatorname{curl} \boldsymbol{\beta}$ in $\Omega_{\mathrm{B}}$, and

$$
\|w\|_{2, \Omega_{\mathrm{B}}}+\|\boldsymbol{\beta}\|_{1, \Omega_{\mathrm{B}}} \leq C_{\mathrm{B}}\left\|\boldsymbol{v}_{\mathrm{B}}\right\|_{\operatorname{div}, \Omega_{\mathrm{B}}} .
$$

Then, we define the discrete Helmholtz decomposition associated to $\boldsymbol{v}_{h}^{\mathrm{B}}$ as

$$
\boldsymbol{v}_{h}^{\mathrm{B}}:=\Pi_{h}^{\mathrm{B}}(\nabla w)+\operatorname{curl}\left(\mathbf{I}_{h}^{\mathrm{B}} \boldsymbol{\beta}\right) \in \mathbf{H}_{h}^{\mathrm{B}},
$$

where $\Pi_{h}^{\mathrm{B}}$ and $\mathbf{I}_{h}^{\mathrm{B}}$ are the Raviart-Thomas and Clément operators, respectively, introduced in Sect. 3.1. Then, using from (3.15) that $\mathbf{E}_{1}\left(\boldsymbol{v}_{h}^{\mathrm{B}}\right)=0$, we can rewrite

$$
\mathbf{E}_{1}\left(\boldsymbol{v}_{\mathrm{B}}\right)=\mathbf{E}_{1}\left(\boldsymbol{v}_{\mathrm{B}}-\boldsymbol{v}_{h}^{\mathrm{B}}\right)=\mathbf{E}_{1}\left(\nabla w-\Pi_{h}^{\mathrm{B}}(\nabla w)\right)+\mathbf{E}_{1}\left(\operatorname{curl}\left(\boldsymbol{\beta}-\mathbf{I}_{h}^{\mathrm{B}} \boldsymbol{\beta}\right)\right) .
$$

Consequently, in what follows we derive suitable upper bounds for the module of the two expressions on the right hand side of (3.17), which are provided by the following two lemmas. 
Lemma 8 There exists $C>0$, independent of meshsizes, such that for each $w \in$ $\mathrm{H}^{2}\left(\Omega_{\mathrm{B}}\right)$ there holds

$$
\left|\mathbf{E}_{1}\left(\nabla w-\Pi_{h}^{\mathrm{B}}(\nabla w)\right)\right| \leq C\left\{\sum_{T \in \mathcal{T}_{h}} \widetilde{\Theta}_{1, T}^{2}\right\}^{\frac{1}{2}}\|w\|_{2, \Omega_{\mathrm{B}}},
$$

where

$$
\widetilde{\Theta}_{1, T}^{2}:=h_{T}^{2}\left\|\boldsymbol{f}_{\mathrm{B}}-\kappa_{\mathrm{B}}^{-1} \boldsymbol{u}_{h}^{\mathrm{B}}-v \operatorname{curl} \boldsymbol{\omega}_{h}^{\mathrm{B}}\right\|_{0, T}^{2}+\sum_{F \in \mathcal{F}_{h, T}(\Sigma)} h_{F}\left\|p_{h}^{\mathrm{B}}-\lambda_{h}\right\|_{0, F}^{2} .
$$

Proof Using the definition of the functional $\mathbf{E}_{1}$ (cf. 3.14), the identity (3.2), the fact that $\left.p_{h}^{\mathrm{B}}\right|_{F} \in P_{0}(F)$ for each $F \in \mathcal{F}_{h}(\Sigma)$, and the characterisation of $\Pi_{h}^{\mathrm{B}}$ given in (3.1), we find that

$$
\begin{aligned}
\mathbf{E}_{1}\left(\nabla w-\Pi_{h}^{\mathrm{B}}(\nabla w)\right)= & \int_{\Omega_{\mathrm{B}}}\left(\boldsymbol{f}_{\mathrm{B}}-\kappa_{\mathrm{B}}^{-1} \boldsymbol{u}_{h}^{\mathrm{B}}-v \operatorname{curl} \boldsymbol{\omega}_{h}^{\mathrm{B}}\right) \cdot\left(\nabla w-\Pi_{h}^{\mathrm{B}}(\nabla w)\right) \\
& +\left\langle\left(\nabla w-\Pi_{h}^{\mathrm{B}}(\nabla w)\right) \cdot \boldsymbol{n}, p_{h}^{\mathrm{B}}-\lambda_{h}\right\rangle_{\Sigma} .
\end{aligned}
$$

In turn, the fact that $\nabla w \in \mathrm{H}^{1}\left(\Omega_{\mathrm{B}}\right)$ guarantees that $\left(\nabla w-\Pi_{h}^{\mathrm{B}}(\nabla w)\right) \cdot \boldsymbol{n} \in \mathrm{L}^{2}(\Sigma)$, and hence

$$
\left\langle\left(\nabla w-\Pi_{h}^{\mathrm{B}}(\nabla w)\right) \cdot \boldsymbol{n}, p_{h}^{\mathrm{B}}-\lambda_{h}\right\rangle_{\Sigma}=\sum_{F \in \mathcal{F}_{h}(\Sigma)} \int_{F}\left(\nabla w-\Pi_{h}^{\mathrm{B}}(\nabla w)\right) \cdot \boldsymbol{n}\left(p_{h}^{\mathrm{B}}-\lambda_{h}\right),
$$

which, together with (3.19), gives

$$
\begin{aligned}
\mathbf{E}_{1}\left(\nabla w-\Pi_{h}^{\mathrm{B}}(\nabla w)\right)= & \sum_{T \in \mathcal{T}_{h}^{\mathrm{B}}} \int_{T}\left(f_{\mathrm{B}}-\kappa_{\mathrm{B}}^{-1} \boldsymbol{u}_{h}^{\mathrm{B}}-v \operatorname{curl} \boldsymbol{\omega}_{h}^{\mathrm{B}}\right) \cdot\left(\nabla w-\Pi_{h}^{\mathrm{B}}(\nabla w)\right) \\
& +\sum_{F \in \mathcal{F}_{h}(\Sigma)} \int_{F}\left(\nabla w-\Pi_{h}^{\mathrm{B}}(\nabla w)\right) \cdot \boldsymbol{n}\left(p_{h}^{\mathrm{B}}-\lambda_{h}\right) .
\end{aligned}
$$

In this way, employing the Cauchy-Schwarz inequality, and the approximation properties of $\Pi_{h}^{\mathrm{B}}$ given in Lemma 1, we deduce from the above expression that

$$
\begin{aligned}
& \left|\mathbf{E}_{1}\left(\nabla w-\Pi_{h}^{\mathrm{B}}(\nabla w)\right)\right| \\
& \leq C\left\{\sum_{T \in \mathcal{T}_{h}^{\mathrm{B}}} h_{T}^{2}\left\|\boldsymbol{f}_{\mathrm{B}}-\kappa_{\mathrm{B}}^{-1} \boldsymbol{u}_{h}^{\mathrm{B}}-v \operatorname{curl} \boldsymbol{\omega}_{h}^{\mathrm{B}}\right\|_{0, T}\right. \\
& \left.\quad+\sum_{F \in \mathcal{F}_{h}(\Sigma)} h_{F}\left\|p_{h}^{\mathrm{B}}-\lambda_{h}\right\|_{0, F}\right\}^{\frac{1}{2}}\|w\|_{2, \Omega_{\mathrm{B}}},
\end{aligned}
$$

which yields (3.18) and completes the proof. 
Lemma 9 There exists $C>0$, independent of meshsizes, such that for each $\boldsymbol{\beta} \in$ $\mathrm{H}^{1}\left(\Omega_{\mathrm{B}}\right)$ there holds

$$
\left|\mathbf{E}_{1}\left(\operatorname{curl}\left(\boldsymbol{\beta}-\mathbf{I}_{h}^{\mathrm{B}} \boldsymbol{\beta}\right)\right)\right| \leq C\left\{\sum_{T \in \mathcal{T}_{h}} \widetilde{\Theta}_{2, T}^{2}\right\}^{\frac{1}{2}}\|\boldsymbol{\beta}\|_{1, \Omega_{\mathrm{B}}}
$$

where

$$
\begin{aligned}
\widetilde{\Theta}_{2, T}^{2}:= & h_{T}^{2}\left\|\operatorname{curl}\left\{\boldsymbol{f}_{\mathrm{B}}-\kappa_{\mathrm{B}}^{-1} \boldsymbol{u}_{h}^{\mathrm{B}}-v \operatorname{curl} \boldsymbol{\omega}_{h}^{\mathrm{B}}\right\}\right\|_{0, T}^{2} \\
& +\sum_{F \in \mathcal{F}_{h, T}\left(\Omega_{\mathrm{B}}\right)} h_{F}\left\|\llbracket\left(\boldsymbol{f}_{\mathrm{B}}-\kappa_{\mathrm{B}}^{-1} \boldsymbol{u}_{h}^{\mathrm{B}}-v \operatorname{curl} \boldsymbol{\omega}_{h}^{\mathrm{B}}\right) \times \boldsymbol{n} \rrbracket\right\|_{0, F}^{2} \\
& +\sum_{F \in \mathcal{F}_{h, T}\left(\Gamma_{\mathrm{B}}\right)} h_{F}\left\|\left(\boldsymbol{f}_{\mathrm{B}}-\kappa_{\mathrm{B}}^{-1} \boldsymbol{u}_{h}^{\mathrm{B}}-v \operatorname{curl} \boldsymbol{\omega}_{h}^{\mathrm{B}}\right) \times \boldsymbol{n}\right\|_{0, F}^{2} \\
& +\sum_{F \in \mathcal{F}_{h, T}(\Sigma)} h_{F}\left\|\left(\boldsymbol{f}_{\mathrm{B}}-\kappa_{\mathrm{B}}^{-1} \boldsymbol{u}_{h}^{\mathrm{B}}-v \operatorname{curl} \boldsymbol{\omega}_{h}^{\mathrm{B}}\right) \times \boldsymbol{n}-\operatorname{curl}_{s}\left(\lambda_{h}\right)\right\|_{0, F}^{2} .
\end{aligned}
$$

Proof Given $\boldsymbol{\beta} \in \mathbf{H}^{1}\left(\Omega_{\mathrm{B}}\right)$, we deduce from (3.14) and the identity $\operatorname{div}\left\{\operatorname{curl}\left(\boldsymbol{\beta}-\mathbf{I}_{h}^{\mathrm{B}} \boldsymbol{\beta}\right)\right\}=0$, that

$$
\begin{aligned}
\mathbf{E}_{1}\left(\operatorname{curl}\left(\boldsymbol{\beta}-\mathbf{I}_{h}^{\mathrm{B}} \boldsymbol{\beta}\right)\right)= & \int_{\Omega_{\mathrm{B}}}\left(\boldsymbol{f}_{\mathrm{B}}-\kappa_{\mathrm{B}}^{-1} \boldsymbol{u}_{h}^{\mathrm{B}}-v \operatorname{curl} \omega_{h}^{\mathrm{B}}\right) \cdot \operatorname{curl}\left(\boldsymbol{\beta}-\mathbf{I}_{h}^{\mathrm{B}} \boldsymbol{\beta}\right) \\
& -\left\langle\operatorname{curl}\left(\boldsymbol{\beta}-\mathbf{I}_{h}^{\mathrm{B}} \boldsymbol{\beta}\right) \cdot \boldsymbol{n}, \lambda_{h}\right\rangle_{\Sigma} .
\end{aligned}
$$

In turn, thanks to the identities given in [30, Chapter I, Eq. (2.17) and Theorem 2.11], we find that

$$
\begin{aligned}
\left\langle\operatorname{curl}\left(\boldsymbol{\beta}-\mathbf{I}_{h}^{\mathrm{B}} \boldsymbol{\beta}\right) \cdot \boldsymbol{n}, \lambda_{h}\right\rangle_{\Sigma} & =\left\langle\operatorname{curl}_{s}\left(\lambda_{h}\right), \boldsymbol{\beta}-\mathbf{I}_{h}^{\mathrm{B}} \boldsymbol{\beta}\right\rangle_{\Sigma} \\
& =\sum_{F \in \mathcal{F}_{h}(\Sigma)} \int_{F} \operatorname{curl}_{s}\left(\lambda_{h}\right) \cdot\left(\boldsymbol{\beta}-\mathbf{I}_{h}^{\mathrm{B}} \boldsymbol{\beta}\right),
\end{aligned}
$$

which gives

$$
\begin{aligned}
\mathbf{E}_{1}\left(\operatorname{curl}\left(\boldsymbol{\beta}-\mathbf{I}_{h}^{\mathrm{B}} \boldsymbol{\beta}\right)\right)= & \sum_{T \in \mathcal{T}_{h}^{\mathrm{B}}} \int_{T}\left(f_{\mathrm{B}}-\kappa_{\mathrm{B}}^{-1} \boldsymbol{u}_{h}^{\mathrm{B}}-v \operatorname{curl} \boldsymbol{\omega}_{h}^{\mathrm{B}}\right) \cdot \operatorname{curl}\left(\boldsymbol{\beta}-\mathbf{I}_{h}^{\mathrm{B}} \boldsymbol{\beta}\right) \\
& -\sum_{F \in \mathcal{F}_{h}(\Sigma)} \int_{F} \operatorname{curl}_{s}\left(\lambda_{h}\right) \cdot\left(\boldsymbol{\beta}-\mathbf{I}_{h}^{\mathrm{B}} \boldsymbol{\beta}\right) .
\end{aligned}
$$

Now, integrating by parts in the first term on the right hand side of the last equation, we obtain 


$$
\begin{aligned}
& \mathbf{E}_{1}\left(\operatorname{curl}\left(\boldsymbol{\beta}-\mathbf{I}_{h}^{\mathrm{B}} \boldsymbol{\beta}\right)\right) \\
& =-\sum_{T \in \mathcal{T}_{h}^{\mathrm{B}}} \int_{T} \operatorname{curl}\left\{\boldsymbol{f}_{\mathrm{B}}-\kappa_{\mathrm{B}}^{-1} \boldsymbol{u}_{h}^{\mathrm{B}}-v \operatorname{curl} \omega_{h}^{\mathrm{B}}\right\} \cdot\left(\boldsymbol{\beta}-\mathbf{I}_{h}^{\mathrm{B}} \boldsymbol{\beta}\right) \\
& \quad+\sum_{F \in \mathcal{F}_{h}\left(\Omega_{\mathrm{B}}\right)} \int_{F} \llbracket\left(\boldsymbol{f}_{\mathrm{B}}-\kappa_{\mathrm{B}}^{-1} \boldsymbol{u}_{h}^{\mathrm{B}}-v \operatorname{curl} \omega_{h}^{\mathrm{B}}\right) \times \boldsymbol{n} \rrbracket \cdot\left(\boldsymbol{\beta}-\mathbf{I}_{h}^{\mathrm{B}} \boldsymbol{\beta}\right) \\
& \quad+\sum_{F \in \mathcal{F}_{h}\left(\Gamma_{\mathrm{B}}\right)} \int_{F}\left\{\left(f_{\mathrm{B}}-\kappa_{\mathrm{B}}^{-1} \boldsymbol{u}_{h}^{\mathrm{B}}-v \operatorname{curl} \omega_{h}^{\mathrm{B}}\right) \times \boldsymbol{n}\right\} \cdot\left(\boldsymbol{\beta}-\mathbf{I}_{h}^{\mathrm{B}} \boldsymbol{\beta}\right) \\
& \quad+\sum_{F \in \mathcal{F}_{h}(\Sigma)} \int_{F}\left\{\left(\boldsymbol{f}_{\mathrm{B}}-\kappa_{\mathrm{B}}^{-1} \boldsymbol{u}_{h}^{\mathrm{B}}-v \operatorname{curl} \omega_{h}^{\mathrm{B}}\right) \times \boldsymbol{n}-\operatorname{curl}_{s}\left(\lambda_{h}\right)\right\} \cdot\left(\boldsymbol{\beta}-\mathbf{I}_{h}^{\mathrm{B}} \boldsymbol{\beta}\right) .
\end{aligned}
$$

Applying Cauchy-Schwarz inequality, Lemma 2, and the uniform boundedness of the number of tetrahedra of the macro-elements $\Delta_{\mathrm{B}}(T)$ and $\Delta_{\mathrm{B}}(F)$, we deduce from (3.21) that

$$
\begin{aligned}
& \left|\mathbf{E}_{1}\left(\operatorname{curl}\left(\boldsymbol{\beta}-\mathbf{I}_{h}^{\mathrm{B}} \boldsymbol{\beta}\right)\right)\right| \\
& \leq \sum_{T \in \mathcal{T}_{h}^{\mathrm{B}}}\left\{h_{T}^{2}\left\|\operatorname{curl}\left\{\boldsymbol{f}_{\mathrm{B}}-\kappa_{\mathrm{B}}^{-1} \boldsymbol{u}_{h}^{\mathrm{B}}-v \operatorname{curl} \boldsymbol{\omega}_{h}^{\mathrm{B}}\right\}\right\|_{0, T}^{2}\right. \\
& \quad+\sum_{F \in \mathcal{F}_{h, T}\left(\Omega_{\mathrm{B}}\right)} h_{F}\left\|\llbracket\left(\boldsymbol{f}_{\mathrm{B}}-\kappa_{\mathrm{B}}^{-1} \boldsymbol{u}_{h}^{\mathrm{B}}-v \operatorname{curl} \boldsymbol{\omega}_{h}^{\mathrm{B}}\right) \times \boldsymbol{n} \rrbracket\right\|_{0, F}^{2} \\
& \quad+\sum_{F \in \mathcal{F}_{h, T}\left(\Gamma_{\mathrm{B}}\right)} h_{F}\left\|\left(\boldsymbol{f}_{\mathrm{B}}-\kappa_{\mathrm{B}}^{-1} \boldsymbol{u}_{h}^{\mathrm{B}}-v \operatorname{curl} \boldsymbol{\omega}_{h}^{\mathrm{B}}\right) \times \boldsymbol{n}\right\|_{0, F}^{2} \\
& \left.\quad+\sum_{F \in \mathcal{F}_{h, T}(\Sigma)} h_{F}\left\|\left(\boldsymbol{f}_{\mathrm{B}}-\kappa_{\mathrm{B}}^{-1} \boldsymbol{u}_{h}^{\mathrm{B}}-v \operatorname{curl} \boldsymbol{\omega}_{h}^{\mathrm{B}}\right) \times \boldsymbol{n}-\operatorname{curl}_{s}\left(\lambda_{h}\right)\right\|_{0, F}^{2}\right\}^{\frac{1}{2}}\|\boldsymbol{\beta}\|_{1, \Omega_{\mathrm{B}}},
\end{aligned}
$$

which implies (3.20) and ends the proof.

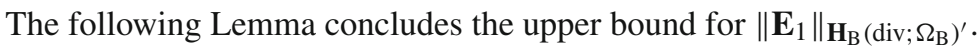

Lemma 10 Assume that there exists a convex domain $\Xi_{\mathrm{B}}$ such that $\Omega_{\mathrm{B}} \subseteq \Xi_{\mathrm{B}}$ and $\Gamma_{\mathrm{B}} \subseteq \partial \Xi_{\mathrm{B}}$. Then, there exists $C_{1}>0$, independent of meshsizes, such that

$$
\left\|\mathbf{E}_{1}\right\|_{\mathbf{H}_{\mathrm{B}}\left(\operatorname{div} ; \Omega_{\mathrm{B}}\right)^{\prime}} \leq C_{1}\left\{\sum_{T \in \mathcal{T}_{h}^{\mathrm{B}}} \widetilde{\Theta}_{\mathrm{B}, T}^{2}\right\}^{\frac{1}{2}},
$$

where $\widetilde{\Theta}_{\mathrm{B}, T}^{2}:=\widetilde{\Theta}_{1, T}^{2}+\widetilde{\Theta}_{2, T}^{2}$, that is

$$
\widetilde{\Theta}_{\mathrm{B}, T}^{2}:=h_{T}^{2}\left\|\boldsymbol{f}_{\mathrm{B}}-\kappa_{\mathrm{B}}^{-1} \boldsymbol{u}_{h}^{\mathrm{B}}-v \operatorname{curl} \omega_{h}^{\mathrm{B}}\right\|_{0, T}^{2}
$$




$$
\begin{aligned}
& +h_{T}^{2}\left\|\operatorname{curl}\left\{\boldsymbol{f}_{\mathrm{B}}-\kappa_{\mathrm{B}}^{-1} \boldsymbol{u}_{h}^{\mathrm{B}}-v \operatorname{curl} \boldsymbol{\omega}_{h}^{\mathrm{B}}\right\}\right\|_{0, T}^{2} \\
& +\sum_{F \in \mathcal{F}_{h, T}\left(\Omega_{\mathrm{B}}\right)} h_{F}\left\|\llbracket\left(\boldsymbol{f}_{\mathrm{B}}-\kappa_{\mathrm{B}}^{-1} \boldsymbol{u}_{h}^{\mathrm{B}}-v \operatorname{curl} \boldsymbol{\omega}_{h}^{\mathrm{B}}\right) \times \boldsymbol{n} \rrbracket\right\|_{0, F}^{2} \\
& +\sum_{F \in \mathcal{F}_{h, T}\left(\Gamma_{\mathrm{B}}\right)} h_{F}\left\|\left(\boldsymbol{f}_{\mathrm{B}}-\kappa_{\mathrm{B}}^{-1} \boldsymbol{u}_{h}^{\mathrm{B}}-v \operatorname{curl} \boldsymbol{\omega}_{h}^{\mathrm{B}}\right) \times \boldsymbol{n}\right\|_{0, F}^{2} \\
& +\sum_{F \in \mathcal{F}_{h, T}(\Sigma)} h_{F}\left\{\left\|\left(\boldsymbol{f}_{\mathrm{B}}-\kappa_{\mathrm{B}}^{-1} \boldsymbol{u}_{h}^{\mathrm{B}}-v \operatorname{curl} \boldsymbol{\omega}_{h}^{\mathrm{B}}\right) \times \boldsymbol{n}-\operatorname{curl}_{s}\left(\lambda_{h}\right)\right\|_{0, F}^{2}\right. \\
& \left.+\left\|p_{h}^{\mathrm{B}}-\lambda_{h}\right\|_{0, F}^{2}\right\} .
\end{aligned}
$$

Proof It follows from (3.18), (3.20), and the stability of the Helmholtz decomposition (3.16).

\subsubsection{Upper bounds for $\left\|\mathbf{E}_{2}\right\|_{\mathbf{H}_{0}\left(\mathbf{c u r l} ; \Omega_{\mathrm{B}}\right)^{\prime}}$ and $\left\|\mathbf{E}_{3}\right\|_{\mathbf{H}_{\mathrm{D}}\left(\operatorname{div} ; \Omega_{\mathrm{D}}\right)^{\prime}}$}

We first establish the upper bound for $\left\|\mathbf{E}_{3}\right\|_{\mathbf{H}_{\mathrm{D}}\left(\mathrm{div} ; \Omega_{\mathrm{D}}\right)^{\prime}}$, which is basically a "mirror reflection" through $\Sigma$ of Lemma 10.

Lemma 11 Assume that there exists a convex domain $\Xi_{\mathrm{D}}$ such that $\Omega_{\mathrm{D}} \subseteq \Xi_{\mathrm{D}}$ and $\Gamma_{\mathrm{D}} \subseteq \partial \Xi_{\mathrm{D}}$. Then, there exists $C_{3}>0$, independent of the meshsizes, such that

$$
\left\|\mathbf{E}_{3}\right\|_{\mathbf{H}_{\mathrm{D}}\left(\operatorname{div} ; \Omega_{\mathrm{D}}\right)^{\prime}} \leq C_{3}\left\{\sum_{T \in \mathcal{T}_{h}^{\mathrm{D}}} \widetilde{\Theta}_{\mathrm{D}, T}^{2}\right\}^{\frac{1}{2}} .
$$

where

$$
\begin{aligned}
\widetilde{\Theta}_{\mathrm{D}, T}^{2}:= & h_{T}^{2}\left\|\boldsymbol{f}_{\mathrm{D}}-\kappa_{\mathrm{D}}^{-1} \boldsymbol{u}_{h}^{\mathrm{D}}\right\|_{0, T}^{2}+h_{T}^{2}\left\|\operatorname{curl}\left\{\boldsymbol{f}_{\mathrm{D}}-\kappa_{\mathrm{D}}^{-1} \boldsymbol{u}_{h}^{\mathrm{D}}\right\}\right\|_{0, T}^{2} \\
& +\sum_{F \in \mathcal{F}_{h, T}\left(\Omega_{\mathrm{B}}\right)} h_{F}\left\|\llbracket\left(\boldsymbol{f}_{\mathrm{D}}-\kappa_{\mathrm{D}}^{-1} \boldsymbol{u}_{h}^{\mathrm{D}}\right) \times \boldsymbol{n} \rrbracket\right\|_{0, F}^{2} \\
& +\sum_{F \in \mathcal{F}_{h, T}\left(\Gamma_{\mathrm{D}}\right)} h_{F}\left\|\left(\boldsymbol{f}_{\mathrm{D}}-\kappa_{\mathrm{D}}^{-1} \boldsymbol{u}_{h}^{\mathrm{D}}\right) \times \boldsymbol{n}\right\|_{0, F}^{2} \\
& +\sum_{F \in \mathcal{F}_{h, T}(\Sigma)} h_{F}\left\{\left\|\left(\boldsymbol{f}_{\mathrm{D}}-\kappa_{\mathrm{D}}^{-1} \boldsymbol{u}_{h}^{\mathrm{D}}\right) \times \boldsymbol{n}-\operatorname{curl}_{S}\left(\lambda_{h}\right)\right\|_{0, F}^{2}+\left\|p_{h}^{\mathrm{D}}-\lambda_{h}\right\|_{0, F}^{2}\right\} .
\end{aligned}
$$

Proof It proceeds exactly as in the proofs of Lemmas 8, 9, and 10, by replacing $\Omega_{\mathrm{B}}$, $\Gamma_{\mathrm{B}}$, and $\mathbf{H}_{\mathrm{B}}\left(\operatorname{div} ; \Omega_{\mathrm{B}}\right)$ by $\Omega_{\mathrm{D}}, \Gamma_{\mathrm{D}}$, and $\mathbf{H}_{\mathrm{D}}\left(\right.$ div; $\left.\Omega_{\mathrm{D}}\right)$, respectively. We omit further details.

The upper bound for $\left\|\mathbf{E}_{2}\right\|_{\mathbf{H}_{0}\left(\mathbf{c u r l} ; \Omega_{\mathrm{B}}\right)^{\prime}}$ is provided next. Indeed, the derivation of this bound hinges on the Helmholtz decomposition given in Lemma 5, integration by parts, and the approximation properties of the Clément operators $I_{h}^{B}$ and $\mathcal{N}_{h}$ established in Lemmas 2 and 3, respectively. 
Lemma 12 There exists $C_{2}>0$, independent of the meshsizes, such that

$$
\left\|\mathbf{E}_{2}\right\|_{\mathbf{H}_{0}\left(\operatorname{curl} ; \Omega_{\mathrm{B}}\right)^{\prime}} \leq C_{2}\left\{\sum_{T \in \mathcal{T}_{h}^{\mathrm{B}}} \widehat{\Theta}_{\mathrm{B}, T}^{2}\right\}^{\frac{1}{2}}
$$

where

$$
\widehat{\Theta}_{\mathrm{B}, T}^{2}:=h_{T}^{2}\left\|\boldsymbol{\omega}_{h}^{\mathrm{B}}-\mathbf{c u r l} \boldsymbol{u}_{h}^{\mathrm{B}}\right\|_{0, T}^{2}+\sum_{F \in \mathcal{F}_{h, T}\left(\Omega_{\mathrm{B}}\right)} h_{F}\left\{\left\|\llbracket \boldsymbol{u}_{h}^{\mathrm{B}} \times \boldsymbol{n} \rrbracket\right\|_{0, F}^{2}+\left\|\llbracket \boldsymbol{\omega}_{h}^{\mathrm{B}} \cdot \boldsymbol{n} \rrbracket\right\|_{0, F}^{2}\right\} .
$$

Proof Given $z_{\mathrm{B}} \in \mathbf{H}_{0}\left(\mathbf{c u r l} ; \Omega_{\mathrm{B}}\right)$, we know from Lemma 5 that there exist $\boldsymbol{\varphi} \in$ $\mathbf{H}_{0}^{1}\left(\Omega_{\mathrm{B}}\right)$ and $\chi \in \mathrm{H}_{0}^{1}\left(\Omega_{\mathrm{B}}\right)$, such that

$$
z_{\mathrm{B}}=\varphi+\nabla \chi \text { in } \Omega_{\mathrm{B}},
$$

and

$$
\|\varphi\|_{1, \Omega_{\mathrm{B}}}+\|\chi\|_{1, \Omega_{\mathrm{B}}} \leq C\left\|z_{\mathrm{B}}\right\|_{\operatorname{curl}, \Omega_{\mathrm{B}}} .
$$

Next, employing the operators $\mathrm{I}_{h}^{\mathrm{B}}$ and $\mathcal{N}_{h}$ defined in Sect. 3.1, we introduce the following discrete Helmholtz decomposition

$$
z_{h}^{\mathrm{B}}:=\mathcal{N}_{h}(\varphi)+\nabla \mathrm{I}_{h}^{\mathrm{B}}(\chi)
$$

which clearly belongs to $\mathbf{H}_{h, 0}^{\mathrm{B}}$. In this way, and recalling from (3.15) that $\mathbf{E}_{2}\left(z_{h}^{\mathrm{B}}\right)=0$, it follows that

$$
\mathbf{E}_{2}\left(z_{\mathrm{B}}\right)=\mathbf{E}_{2}\left(z_{\mathrm{B}}-z_{h}^{\mathrm{B}}\right)=\mathbf{E}_{2}\left(\boldsymbol{\varphi}-\mathcal{N}_{h}(\boldsymbol{\varphi})\right)+\mathbf{E}_{2}\left(\nabla\left(\chi-\mathrm{I}_{h}^{\mathrm{B}}(\chi)\right)\right),
$$

from which, according to the definition of $\mathbf{E}_{2}$ (cf. 3.14), we find that

$$
\begin{aligned}
\mathbf{E}_{2}\left(z_{\mathrm{B}}\right)= & v \int_{\Omega_{\mathrm{B}}} \boldsymbol{u}_{h}^{\mathrm{B}} \cdot \operatorname{curl}\left(\boldsymbol{\varphi}-\mathcal{N}_{h}(\boldsymbol{\varphi})\right)-v \int_{\Omega_{\mathrm{B}}} \boldsymbol{\omega}_{h}^{\mathrm{B}} \cdot\left(\boldsymbol{\varphi}-\mathcal{N}_{h}(\boldsymbol{\varphi})\right) \\
& -v \int_{\Omega_{\mathrm{B}}} \omega_{h}^{\mathrm{B}} \cdot \nabla\left(\chi-\mathrm{I}_{h}^{\mathrm{B}}(\chi)\right) .
\end{aligned}
$$

Then, integrating by parts on each $T$, and noting that $\operatorname{div} \omega_{h}^{\mathrm{B}}$ is zero on $T$ (cf. 2.6, 2.7), we have

$$
\begin{gathered}
\mathbf{E}_{2}\left(z_{\mathrm{B}}\right)=\sum_{T \in \mathcal{T}_{h}^{\mathrm{B}}} v \int_{T} \operatorname{curl} \boldsymbol{u}_{h}^{\mathrm{B}} \cdot\left(\boldsymbol{\varphi}-\mathcal{N}_{h}(\boldsymbol{\varphi})\right)-\sum_{T \in \mathcal{T}_{h}^{\mathrm{B}}} v \int_{T} \boldsymbol{\omega}_{h}^{\mathrm{B}} \cdot\left(\boldsymbol{\varphi}-\mathcal{N}_{h}(\boldsymbol{\varphi})\right) \\
-\sum_{F \in \mathcal{F}_{h}\left(\Omega_{\mathrm{B}}\right)} \int_{F} \llbracket \boldsymbol{u}_{h}^{\mathrm{B}} \times \boldsymbol{n} \rrbracket \cdot\left(\boldsymbol{\varphi}-\mathcal{N}_{h}(\boldsymbol{\varphi})\right)-\sum_{F \in \mathcal{F}_{h}\left(\Omega_{\mathrm{B}}\right)} \int_{F} \llbracket \boldsymbol{\omega}_{h}^{\mathrm{B}} \cdot \boldsymbol{n} \rrbracket \cdot\left(\chi-\mathrm{I}_{h}^{\mathrm{B}}(\chi)\right) .
\end{gathered}
$$


In this way, applying Cauchy-Schwarz inequality, the approximation properties of $\mathrm{I}_{h}^{\mathrm{B}}$ and $\mathcal{N}_{h}$ given in Lemmas 2 and 3, respectively, the fact that the number of tetrahedra of the macro-elements $\Delta_{\mathrm{B}}(T)$ and $\Delta_{\mathrm{B}}(F)$ is uniformly bounded, and the stability estimate (3.23), we get (3.22) and finish the proof.

We end this section by concluding that the reliability of $\Theta$, that is the upper bound in (3.8), is a straightforward consequence of Theorem 2 and Lemmas 7, 10-12.

\subsection{Efficiency}

We now devote our attention to the derivation of upper bounds depending on the actual errors associated to the local indicators on each subdomain. For clarity of the analysis we will restrict ourselves to piecewise polynomial forcing terms $f_{\mathrm{B}}$ and $f_{\mathrm{D}}$, but we remark that if they are otherwise sufficiently smooth, the error committed from suitable polynomial approximation would produce additional higher order terms in (3.8), explaining the eventual appearance of h.o.t in that inequality.

First, and thanks to the incompressibility condition in $\Omega_{\mathrm{B}}$ (respectively $\Omega_{\mathrm{D}}$ ), one has that

$$
\left\|\operatorname{div} \boldsymbol{u}_{h}^{\mathrm{B}}\right\|_{0, T} \leq\left\|\boldsymbol{u}_{\mathrm{B}}-\boldsymbol{u}_{h}^{\mathrm{B}}\right\|_{\operatorname{div}, T} \quad \text { and } \quad\left\|\operatorname{div} \boldsymbol{u}_{h}^{\mathrm{D}}\right\|_{0, T} \leq\left\|\boldsymbol{u}_{\mathrm{D}}-\boldsymbol{u}_{h}^{\mathrm{D}}\right\|_{\operatorname{div}, T}
$$

The remaining terms in $\Theta_{\mathrm{B}, T}^{2}$ and $\Theta_{\mathrm{D}, T}^{2}$ can be treated very much in the same way as done in [24,27,28], where the analysis is based on inverse inequalities found in [19], together with the localisation technique based on tetrahedron-bubble and facetbubble functions [34]. Such a theory requires further notation and preliminary results collected in what follows.

Given $T \in \mathcal{T}_{h}$ and $F \in \mathcal{F}(T)$, let $\psi_{T}$ and $\psi_{F}$ denote tetrahedron-bubble and face-bubble functions, respectively (see [33, Eqs. (1.4) and (1.6)]), which satisfy:

(i) $\psi_{T} \in P_{4}(T), \operatorname{supp}\left(\psi_{T}\right) \subseteq T, \psi_{T}=0$ on $\partial T$, and $0 \leq \psi_{T} \leq 1$ in $T$.

(ii) $\left.\psi_{F}\right|_{T} \in P_{3}(T), \operatorname{supp}\left(\psi_{F}\right) \subseteq \omega_{F}:=\cup\left\{T^{\prime} \in \mathcal{T}_{h}: F \in \overline{\mathcal{F}}\left(T^{\prime}\right)\right\}, \psi_{F}=0$ on $\partial T \backslash\{F\}$, and $0 \leq \psi_{F} \leq 1$ in $\omega_{F}$.

In addition, there exists an extension operator $L: C(F) \rightarrow C(T)$ that satisfies $L(p) \in P_{k}(T)$ and $\left.L(p)\right|_{F}=p \forall p \in P_{k}(F)$, for a given $k \geq 0$ (see [32]). The vectorial counterpart of $L$ will be denoted $\mathbf{L}$. Moreover, the following properties hold (where a proof can be found in [32, Lemma 4.1]).

Lemma 13 Given $k \in \mathbb{N} \cup\{0\}$, there exist $c_{1}, c_{2}, c_{3}>0$, depending only on $k$ and the shape regularity of the triangulations, such that for each $T \in \mathcal{T}_{h}$ and $F \in \mathcal{F}(T)$, there hold

$$
\begin{aligned}
&\|q\|_{0, T}^{2} \leq c_{1}\left\|\psi_{T}^{1 / 2} q\right\|_{0, T}^{2} \forall q \in P_{k}(T), \\
&\|p\|_{0, F}^{2} \leq c_{2}\left\|\psi_{F}^{1 / 2} p\right\|_{0, F}^{2} \forall p \in P_{k}(F), \\
&\left\|\psi_{F}^{1 / 2} L(p)\right\|_{0, T}^{2} \leq c_{3} h_{F}\|p\|_{0, F}^{2} \quad \forall p \in P_{k}(F) .
\end{aligned}
$$


The following inverse estimate is also required (see a proof in [19, Theorem 3.2.6]).

Lemma 14 Let $l, m \in \mathbb{N} \cup\{0\}$ such that $l \leq m$. Then, there exists $c_{4}>0$, depending only on $k, l, m$ and the shape regularity of the triangulations, such that for each $T \in \mathcal{T}_{h}$ there holds

$$
|q|_{m, T} \leq c_{4} h_{T}^{l-m}|q|_{l, T} \quad \forall q \in P_{k}(T) .
$$

Finally we give two technical lemmas before tackling the derivation of the required upper bounds.

Lemma 15 Let $\zeta_{h} \in \mathbf{L}^{2}(\Omega)$ be an element-wise polynomial of degree $k \geq 0$, and let $\zeta \in \mathbf{L}^{2}(\Omega)$ be such that $\operatorname{curl}(\zeta)=\mathbf{0}$ in $\Omega$. Then, there exist $c_{5}, c_{6}>0$, independent of the meshsize, such that

$$
\begin{aligned}
h_{T}^{2}\left\|\operatorname{curl}\left(\zeta_{h}\right)\right\|_{0, T}^{2} \leq c_{5}\left\|\zeta-\zeta_{h}\right\|_{0, T}^{2} & \forall T \in \mathcal{T}_{h}, \\
h_{F}\left\|\llbracket \zeta_{h} \times \boldsymbol{n} \rrbracket\right\|_{0, F}^{2} \leq c_{6}\left\|\zeta-\zeta_{h}\right\|_{0, \omega_{F}}^{2} & \forall F \in \mathcal{F}_{h}(\Omega),
\end{aligned}
$$

where the set $\omega_{F}$ is given by $\omega_{F}:=\cup\left\{T^{\prime} \in \mathcal{T}_{h}: F \in \mathcal{F}\left(T^{\prime}\right)\right\}$.

Proof See [24, Lemmas 4.9 and 4.10, respectively].

Lemma 16 Let $\zeta_{h} \in \mathbf{L}^{2}(\Omega)$ be an element-wise polynomial of degree $k \geq 0$, and let $\zeta \in \mathbf{L}^{2}(\Omega)$ be such that $\operatorname{div}(\zeta)=0$ in $\Omega$. Then, there exist $c_{7}, c_{8}>0$, independent of the meshsize, such that

$$
\begin{aligned}
h_{T}^{2}\left\|\operatorname{div}\left(\boldsymbol{\zeta}_{h}\right)\right\|_{0, F}^{2} \leq c_{7}\left\|\zeta-\boldsymbol{\zeta}_{h}\right\|_{0, T}^{2} & \forall T \in \mathcal{T}_{h}, \\
h_{F}\left\|\llbracket \zeta_{h} \cdot \boldsymbol{n} \rrbracket\right\|_{0, F}^{2} \leq c_{8}\left\|\zeta-\zeta_{h}\right\|_{0, \omega_{F}}^{2} & \forall F \in \mathcal{F}_{h}(\Omega) .
\end{aligned}
$$

Proof Indeed, applying the first inequality given in (3.25), using that $\operatorname{div}(\zeta)=0$ in $\Omega$, integrating by parts, and then employing the Cauchy-Schwarz inequality, we get

$$
\begin{aligned}
& \left\|\operatorname{div}\left(\zeta_{h}\right)\right\|_{0, T}^{2} \leq c_{1}\left\|\psi_{T}^{1 / 2} \operatorname{div}\left(\zeta_{h}\right)\right\|_{0, T}^{2}=c_{1} \int_{T} \psi_{T} \operatorname{div}\left(\zeta_{h}\right) \cdot \operatorname{div}\left(\zeta_{h}-\zeta\right) \\
& \leq c_{1} \int_{T}\left(\zeta-\zeta_{h}\right) \cdot \nabla\left(\psi_{T} \operatorname{div}\left(\zeta_{h}\right)\right) \leq\left\|\zeta-\zeta_{h}\right\|_{0, T}\left\|\nabla\left(\psi_{T} \operatorname{div}\left(\zeta_{h}\right)\right)\right\|_{0, T} .
\end{aligned}
$$

Now, using the inverse inequality (3.26), and the fact that $0 \leq \psi_{T} \leq 1$ in $T$, we find that

$$
\left\|\nabla\left(\psi_{T} \operatorname{div}\left(\zeta_{h}\right)\right)\right\|_{0, T} \leq c h_{T}^{-1}\left\|\psi_{T} \operatorname{div}\left(\zeta_{h}\right)\right\|_{0, T} \leq c h_{T}^{-1}\left\|\operatorname{div}\left(\zeta_{h}\right)\right\|_{0, T},
$$

which together with (3.31) gives (3.29). The proof of (3.30) corresponds to a slight adaptation of the proof of [7, Lemma 4.6], which makes use of (3.29).

After these preliminary results, we are ready to give local efficiency estimates for several terms associated to the interface. 
Lemma 17 There exist constants $c_{i}>0, i \in\{9,10,11\}$, independent of the meshsizes, such that

(a) $h_{F}\left\|p_{h}^{\mathrm{B}}-\lambda_{h}\right\|_{0, F}^{2} \leq c_{9}\left\{\left\|p_{\mathrm{B}}-p_{h}^{\mathrm{B}}\right\|_{0, T_{F}}^{2}+h_{T}^{2}\left\|\boldsymbol{u}_{\mathrm{B}}-\boldsymbol{u}_{h}^{\mathrm{B}}\right\|_{0, T_{F}}^{2}+h_{F}\left\|\lambda-\lambda_{h}\right\|_{0, F}^{2}\right\}$, for all $F \in \mathcal{F}_{h}(\Sigma)$, where $T_{F}$ is the tetrahedron of $\mathcal{T}_{h}^{\mathrm{B}}$ having $F$ as a face,

(b) $h_{F}\left\|p_{h}^{\mathrm{D}}-\lambda_{h}\right\|_{0, F}^{2} \leq c_{10}\left\{\left\|p_{\mathrm{D}}-p_{h}^{\mathrm{D}}\right\|_{0, T_{F}}^{2}+h_{T}^{2}\left\|\boldsymbol{u}_{\mathrm{D}}-\boldsymbol{u}_{h}^{\mathrm{D}}\right\|_{0, T_{F}}^{2}+h_{F}\left\|\lambda-\lambda_{h}\right\|_{0, F}^{2}\right\}$, for all $F \in \mathcal{F}_{h}(\Sigma)$, where $T_{F}$ is the tetrahedron of $\mathcal{T}_{h}^{\mathrm{D}}$ having $F$ as a face,

(c) $h_{F}\left\|\boldsymbol{u}_{\mathrm{B}} \cdot \boldsymbol{n}-\boldsymbol{u}_{\mathrm{D}} \cdot \boldsymbol{n}\right\|_{0, F}^{2} \leq c_{11}\left\{\left\|\boldsymbol{u}_{\mathrm{B}}-\boldsymbol{u}_{h}^{\mathrm{B}}\right\|_{0, T_{F}}^{2}+h_{T}^{2}\left\|\operatorname{div}\left(\boldsymbol{u}_{\mathrm{B}}-\boldsymbol{u}_{h}^{\mathrm{B}}\right)\right\|_{0, T_{F}}^{2}\right.$ $\left.+\left\|\boldsymbol{u}_{\mathrm{D}}-\boldsymbol{u}_{h}^{\mathrm{D}}\right\|_{0, T_{F}}^{2}+h_{T}^{2}\left\|\operatorname{div}\left(\boldsymbol{u}_{\mathrm{D}}-\boldsymbol{u}_{h}^{\mathrm{D}}\right)\right\|_{0, T_{F}}^{2}\right\}$,

for all $F \in \mathcal{F}_{h}(\Sigma)$, where $T_{F}$ is the tetrahedron of $\mathcal{T}_{h}^{\mathrm{B}} \cup \mathcal{T}_{h}^{\mathrm{D}}$ having $F$ as a face.

Proof Estimates (a) and (b) can be obtained by adapting the proof of [6, Lemma 4.12], whereas (c) follows after a slight modification of the proof in [27, Lemma 3.17] (see also [6, Lemma 4.7]).

Lemma 18 There exist constants $c_{i}>0, i \in\{12,13\}$, independent of the meshsizes, such that

(a)

$$
\begin{aligned}
& \sum_{F \in \mathcal{F}_{h}(\Sigma)} h_{F}\left\|\left(\boldsymbol{f}_{\mathrm{B}}-\kappa_{\mathrm{B}}^{-1} \boldsymbol{u}_{h}^{\mathrm{B}}-v \operatorname{curl} \boldsymbol{\omega}_{h}^{\mathrm{B}}\right) \times \boldsymbol{n}-\operatorname{curl}_{S}\left(\lambda_{h}\right)\right\|_{0, F}^{2} \\
& \leq c_{12}\left\{\sum_{F \in \mathcal{F}_{h}(\Sigma)}\left(\left\|\boldsymbol{u}_{\mathrm{B}}-\boldsymbol{u}_{h}^{\mathrm{B}}\right\|_{0, T_{F}}^{2}+\left\|\operatorname{curl}\left(\boldsymbol{\omega}_{\mathrm{B}}-\boldsymbol{\omega}_{h}^{\mathrm{B}}\right)\right\|_{0, T_{F}}^{2}\right)\right. \\
& \left.\quad+\left\|\lambda-\lambda_{h}\right\|_{1 / 2, \Sigma}^{2}\right\},
\end{aligned}
$$

(b)

$$
\begin{aligned}
& \sum_{F \in \mathcal{F}_{h}(\Sigma)} h_{F}\left\|\left(\boldsymbol{f}_{\mathrm{D}}-\kappa_{\mathrm{D}}^{-1} \boldsymbol{u}_{h}^{\mathrm{D}}\right) \times \boldsymbol{n}-\operatorname{curl}_{s}\left(\lambda_{h}\right)\right\|_{0, F}^{2} \\
& \leq c_{13}\left\{\sum_{F \in \mathcal{F}_{h}(\Sigma)} \| \boldsymbol{u}_{\mathrm{D}}-\boldsymbol{u}_{h}^{\left.\mathrm{D}_{1}\left\|_{0, T_{F}}^{2}+\right\| \lambda-\lambda_{h} \|_{1 / 2, \Sigma}^{2}\right\} .}\right.
\end{aligned}
$$

where, given $F \in \mathcal{F}_{h}(\Sigma), T_{F}$ is the tetrahedron of $\mathcal{T}_{h}^{\mathrm{B}}$ (respectively $\mathcal{T}_{h}^{\mathrm{D}}$ ) having $F$ as a face.

Proof The proofs of (a) and (b) follow after a straightforward adaptation of that of [25, Lemma 20], and recalling from [13, Lemma 3.6] that the operator $\operatorname{curl}_{s}$ is bounded.

We remark that estimates (a) and (b) provided by the previous lemma are the only nonlocal bounds of the efficiency analysis. However, under an additional regularity assumption on $\lambda$ we are able to prove the following local bounds. 
Lemma 19 Assume that $\left.\lambda\right|_{F} \in \mathrm{H}^{1}(F)$, for each $F \in \mathcal{F}_{h}(\Sigma)$. Then there exist $c_{14}, c_{15}>0$, independent of the meshsizes, such that for each $F \in \mathcal{F}_{h}(\Sigma)$ there hold

$$
\begin{aligned}
& h_{F}\left\|\left(\boldsymbol{f}_{\mathrm{B}}-\kappa_{\mathrm{B}}^{-1} \boldsymbol{u}_{h}^{\mathrm{B}}-v \operatorname{curl} \boldsymbol{\omega}_{h}^{\mathrm{B}}\right) \times \boldsymbol{n}-\operatorname{curl}_{s}\left(\lambda_{h}\right)\right\|_{0, F}^{2} \\
& \quad \leq c_{14}\left\{\left\|\boldsymbol{u}_{\mathrm{B}}-\boldsymbol{u}_{h}^{\mathrm{B}}\right\|_{0, T_{F}}^{2}+\left\|\operatorname{curl}\left(\boldsymbol{\omega}_{\mathrm{B}}-\boldsymbol{\omega}_{h}^{\mathrm{B}}\right)\right\|_{0, T_{F}}^{2}+h_{F}\left\|\operatorname{curl}_{s}\left(\lambda-\lambda_{h}\right)\right\|_{0, F}^{2}\right\}
\end{aligned}
$$

and

$$
\begin{aligned}
& h_{F}\left\|\left(\boldsymbol{f}_{\mathrm{D}}-\kappa_{\mathrm{D}}^{-1} \boldsymbol{u}_{h}^{\mathrm{D}}\right) \times \boldsymbol{n}-\operatorname{curl}_{s}\left(\lambda_{h}\right)\right\|_{0, F}^{2} \\
& \quad \leq c_{15}\left\{\left\|\boldsymbol{u}_{\mathrm{D}}-\boldsymbol{u}_{h}^{\mathrm{D}}\right\|_{0, T_{F}}^{2}+h_{F}\left\|\operatorname{curl}_{s}\left(\lambda-\lambda_{h}\right)\right\|_{0, F}^{2}\right\},
\end{aligned}
$$

where $T_{F}$ is the tetrahedron of $\mathcal{T}_{h}^{\mathrm{B}}$ (respectively $\mathcal{T}_{h}^{\mathrm{D}}$ ) having $F$ as a face.

Proof The derivation of these estimates follows as in the proof of [25, Lemma 21].

The following three lemmas provide the corresponding upper bounds for the remaining terms defining $\Theta_{\mathrm{B}, T}^{2}$ (cf. 3.5) and $\Theta_{\mathrm{D}, T}^{2}$ ( cf. 3.6).

Lemma 20 There exist positive constants $c_{i}, i \in\{16,17,18,19\}$, independent of the meshsizes, such that

(a) $h_{T}^{2}\left\|\operatorname{curl}\left\{\boldsymbol{f}_{\mathrm{B}}-\kappa_{\mathrm{B}}^{-1} \boldsymbol{u}_{h}^{\mathrm{B}}-v \operatorname{curl} \omega_{h}^{\mathrm{B}}\right\}\right\|_{0, T}^{2} \leq c_{16}\left\{\left\|\boldsymbol{u}_{\mathrm{B}}-\boldsymbol{u}_{h}^{\mathrm{B}}\right\|_{0, T}^{2}\right.$ $\left.+\left\|\operatorname{curl}\left(\omega_{\mathrm{B}}-\omega_{h}^{\mathrm{B}}\right)\right\|_{0, T}^{2}\right\}$, for all $T \in \mathcal{T}_{h}^{\mathrm{B}}$.

(b) $\left\|\boldsymbol{f}_{\mathrm{B}}-\kappa_{\mathrm{B}}^{-1} \boldsymbol{u}_{h}^{\mathrm{B}}-v \operatorname{curl} \boldsymbol{\omega}_{h}^{\mathrm{B}}\right\|_{0, T}^{2} \leq c_{17}\left\{\left\|\boldsymbol{u}_{\mathrm{B}}-\boldsymbol{u}_{h}^{\mathrm{B}}\right\|_{0, T}^{2}+\left\|\operatorname{curl}\left(\boldsymbol{\omega}_{\mathrm{B}}-\boldsymbol{\omega}_{h}^{\mathrm{B}}\right)\right\|_{0, T}^{2}+\right.$ $\left.h_{T}^{-2}\left\|p_{\mathrm{B}}-p_{h}^{\mathrm{B}}\right\|_{0, T}^{2}\right\}$, for all $T \in \mathcal{T}_{h}^{\mathrm{B}}$.

(c) $h_{F}\left\|\llbracket\left(f_{\mathrm{B}}-\kappa_{\mathrm{B}}^{-1} \boldsymbol{u}_{h}^{\mathrm{B}}-v \operatorname{curl} \boldsymbol{\omega}_{h}^{\mathrm{B}}\right) \times \boldsymbol{n} \rrbracket\right\|_{0, F}^{2} \leq c_{18}\left\{\left\|\boldsymbol{u}_{\mathrm{B}}-\boldsymbol{u}_{h}^{\mathrm{B}}\right\|_{0, \omega_{F}}^{2}\right.$ $\left.+\left\|\operatorname{curl}\left(\omega_{\mathrm{B}}-\omega_{h}^{\mathrm{B}}\right)\right\|_{0, \omega_{F}}^{2}\right\}$, for all $F \in \mathcal{F}_{h}\left(\Omega_{\mathrm{B}}\right)$, where $\omega_{F}:=\cup\left\{T^{\prime} \in \mathcal{T}_{h}^{\mathrm{B}}:\right.$ $\left.F \in \mathcal{F}\left(T^{\prime}\right)\right\}$.

(d) $h_{F}\left\|\left(f_{\mathrm{B}}-\kappa_{\mathrm{B}}^{-1} \boldsymbol{u}_{h}^{\mathrm{B}}-v \operatorname{curl} \boldsymbol{\omega}_{h}^{\mathrm{B}}\right) \times \boldsymbol{n}\right\|_{0, F}^{2} \leq c_{19}\left\{\left\|\boldsymbol{u}_{\mathrm{B}}-\boldsymbol{u}_{h}^{\mathrm{B}}\right\|_{0, T_{F}}^{2}\right.$ $+\left\|\operatorname{curl}\left(\omega_{\mathrm{B}}-\omega_{h}^{\mathrm{B}}\right)\right\|_{0, T_{F}}^{2}+$ h.o.t $\}$, for all $F \in \mathcal{F}_{h}\left(\Gamma_{\mathrm{B}}\right)$, where $T_{F}$ is the tetrahedron of $\mathcal{T}_{h}^{\mathrm{B}}$ having $F$ as a face, and

$$
\text { h.o.t }:=h_{T_{F}}^{4}\left\|\boldsymbol{u}_{\mathrm{B}}-\boldsymbol{u}_{h}^{\mathrm{B}}\right\|_{0, T_{F}}^{2}+h_{T_{F}}^{4}\left\|\operatorname{curl}\left(\boldsymbol{\omega}_{\mathrm{B}}-\boldsymbol{\omega}_{h}^{\mathrm{B}}\right)\right\|_{0, T_{F}}^{2}+h_{T_{F}}^{2}\left\|p_{\mathrm{B}}-p_{h}^{\mathrm{B}}\right\|_{0, T_{F}}^{2} .
$$

Proof Since $\operatorname{curl}\left(\boldsymbol{f}_{\mathrm{B}}-\kappa_{\mathrm{B}}^{-1} \boldsymbol{u}_{\mathrm{B}}-v \operatorname{curl} \boldsymbol{\omega}_{\mathrm{B}}\right)=\operatorname{curl}\left(\nabla p_{\mathrm{B}}\right)=\mathbf{0}$ in $\Omega_{\mathrm{B}}$, to derive (a) and (c) it suffices to apply the estimates (3.27) and (3.28) (cf. Lemma 15), respectively, to

$$
\zeta:=f_{\mathrm{B}}-\kappa_{\mathrm{B}}^{-1} \boldsymbol{u}_{\mathrm{B}}-v \operatorname{curl} \omega_{\mathrm{B}} \text { and } \zeta_{h}:=f_{\mathrm{B}}-\kappa_{\mathrm{B}}^{-1} \boldsymbol{u}_{h}^{\mathrm{B}}-v \operatorname{curl} \omega_{h}^{\mathrm{B}} .
$$


On the other hand, reasoning similarly as in the proof of [29, Lemma 5.14], we get

$$
\begin{aligned}
\left\|\boldsymbol{\zeta}_{h}\right\|_{0, T} & =\left\|\boldsymbol{f}_{\mathrm{B}}-\kappa_{\mathrm{B}}^{-1} \boldsymbol{u}_{h}^{\mathrm{B}}-v \operatorname{curl} \boldsymbol{\omega}_{h}^{\mathrm{B}}\right\|_{0, T} \\
& \leq c\left\{\left\|\boldsymbol{u}_{\mathrm{B}}-\boldsymbol{u}_{h}^{\mathrm{B}}\right\|_{0, T}+\left\|\operatorname{curl} \boldsymbol{\omega}_{\mathrm{B}}-\boldsymbol{\omega}_{h}^{\mathrm{B}}\right\|_{0, T}+h_{T}^{-1}\left\|p_{\mathrm{B}}-p_{h}^{\mathrm{B}}\right\|_{0, T}\right\},
\end{aligned}
$$

from which, it is easy to deduce the estimate (b). For the proof of (d), we set $\zeta$ and $\zeta_{h}$ as in (3.32). Given $F \in \mathcal{F}_{h}(\Gamma)$ we denote $\chi_{F}:=\boldsymbol{\zeta}_{h} \times \boldsymbol{n}$ on $F$. Then, applying the second inequality given in (3.25), and the extension operator $\mathbf{L}: \mathbf{C}(F) \rightarrow \mathbf{C}(T)$, we find that

$$
\begin{aligned}
\left\|\boldsymbol{\chi}_{F}\right\|_{0, F}^{2} \leq c_{2}\left\|\psi_{F}^{1 / 2} \chi_{F}\right\|_{0, F}^{2} & =c_{2} \int_{F} \psi_{F} \chi_{F} \cdot\left(\boldsymbol{\zeta}_{h} \times \boldsymbol{n}\right) \\
& =c_{2} \int_{\partial T_{F}} \psi_{F} \mathbf{L}\left(\boldsymbol{\chi}_{F}\right) \cdot\left(\boldsymbol{\zeta}_{h} \times \boldsymbol{n}\right) .
\end{aligned}
$$

Now, integrating by parts, it follows that

$\int_{\partial T_{F}} \psi_{F} \mathbf{L}\left(\chi_{F}\right) \cdot\left(\zeta_{h} \times \boldsymbol{n}\right)=\int_{T_{F}} \zeta_{h} \cdot \operatorname{curl}\left(\psi_{F} \mathbf{L}\left(\chi_{F}\right)\right)+\int_{T_{F}} \operatorname{curl}\left(\zeta_{h}\right) \cdot \psi_{F} \mathbf{L}\left(\chi_{F}\right)$

Next, applying the Cauchy-Schwarz inequality, the inverse estimate (3.26), and the preliminary bound for $\left\|\operatorname{curl}\left(\zeta_{h}\right)\right\|_{0, T_{F}}$ (cf. 3.27), we deduce that

$$
\left\|\chi_{F}\right\|_{0, F}^{2} \leq c_{2}\left\{c_{4}\left\|\zeta_{h}\right\|_{0, T_{F}}+\left\|\zeta-\zeta_{h}\right\|_{0, T_{F}}\right\} h_{T_{F}}^{-1}\left\|\psi_{F} \mathbf{L}\left(\chi_{F}\right)\right\|_{0, T_{F}}
$$

In turn, recalling that $0 \leq \psi_{F} \leq 1$ in $F$, and employing the third inequality in (3.25), we can write

$$
\left\|\psi_{F} \mathbf{L}\left(\chi_{F}\right)\right\|_{0, T_{F}} \leq\left\|\psi_{F}^{1 / 2} \mathbf{L}\left(\chi_{F}\right)\right\|_{0, T_{F}} \leq c_{3}^{1 / 2} h_{F}^{1 / 2}\left\|\chi_{F}\right\|_{0, T_{F}} .
$$

Finally, using (3.35), the definitions of $\zeta$ and $\zeta_{h}$ (cf. 3.32), the preliminary estimate (3.33), and the fact that $h_{F} \leq h_{T_{F}}$, we deduce from (3.34) that

$$
h_{F}^{1 / 2}\left\|\chi_{F}\right\|_{0, F} \leq c\left\{\left\|\boldsymbol{u}_{\mathrm{B}}-\boldsymbol{u}_{h}^{\mathrm{B}}\right\|_{0, T_{F}}+\left\|\operatorname{curl}\left(\boldsymbol{\omega}_{\mathrm{B}}-\boldsymbol{\omega}_{h}^{\mathrm{B}}\right)\right\|_{0, T_{F}}+\text { h.o.t }\right\},
$$

where

$$
\text { h.o.t }:=h_{T_{F}}^{2}\left\|\boldsymbol{u}_{\mathrm{B}}-\boldsymbol{u}_{h}^{\mathrm{B}}\right\|_{0, T_{F}}+h_{T_{F}}^{2}\left\|\operatorname{curl}\left(\boldsymbol{\omega}_{\mathrm{B}}-\boldsymbol{\omega}_{h}^{\mathrm{B}}\right)\right\|_{0, T_{F}}+h_{T_{F}}\left\|p_{\mathrm{B}}-p_{h}^{\mathrm{B}}\right\|_{0, T_{F}},
$$

which gives (d), and ends the proof.

Lemma 21 There exist $c_{i}>0, i \in\{20,21,22,23\}$, independent of the meshsizes, such that 
(a) $h_{T}^{2}\left\|\operatorname{curl}\left\{\boldsymbol{f}_{\mathrm{D}}-\kappa_{\mathrm{D}}^{-1} \boldsymbol{u}_{h}^{\mathrm{D}}\right\}\right\|_{0, T}^{2} \leq c_{20}\left\|\boldsymbol{u}_{\mathrm{D}}-\boldsymbol{u}_{h}^{\mathrm{D}}\right\|_{0, T}^{2} \quad \forall T \in \mathcal{T}_{h}^{\mathrm{D}}$,

(b) $h_{T}^{2}\left\|\boldsymbol{f}_{\mathrm{D}}-\kappa_{\mathrm{D}}^{-1} \boldsymbol{u}_{h}^{\mathrm{D}}\right\|_{0, T}^{2} \leq c_{21}\left\{h_{T}^{2}\left\|\boldsymbol{u}_{\mathrm{D}}-\boldsymbol{u}_{h}^{\mathrm{D}}\right\|_{0, T}^{2}+\left\|p_{\mathrm{D}}-p_{h}^{\mathrm{D}}\right\|_{0, T}^{2}\right\} \quad \forall T \in \mathcal{T}_{h}^{\mathrm{D}}$,

(c) $h_{F}\left\|\llbracket\left(\boldsymbol{f}_{\mathrm{D}}-\kappa_{\mathrm{D}}^{-1} \boldsymbol{u}_{h}^{\mathrm{D}}\right) \times \boldsymbol{n} \rrbracket\right\|_{0, F}^{2} \leq c_{22}\left\|\boldsymbol{u}_{\mathrm{D}}-\boldsymbol{u}_{h}^{\mathrm{D}}\right\|_{0, \omega_{F}}^{2}$ for all $F \in \mathcal{F}_{h}\left(\Omega_{\mathrm{D}}\right)$, where the set $\omega_{F}$ is given by $\omega_{F}:=\cup\left\{T^{\prime} \in \mathcal{T}_{h}^{\mathrm{D}}: F \in \mathcal{F}\left(T^{\prime}\right)\right\}$,

(d) $h_{F}\left\|\left(\boldsymbol{f}_{\mathrm{D}}-\kappa_{\mathrm{D}}^{-1} \boldsymbol{u}_{h}^{\mathrm{D}}\right) \times \boldsymbol{n}\right\|_{0, F}^{2} \leq c_{23}\left\{\left\|\boldsymbol{u}_{\mathrm{D}}-\boldsymbol{u}_{h}^{\mathrm{D}}\right\|_{0, T_{F}}^{2}+\left\|p_{\mathrm{D}}-p_{h}^{\mathrm{D}}\right\|_{0, T_{F}}^{2}+\right.$ h.o.t $\}$ for all $F \in \mathcal{F}_{h}\left(\Gamma_{\mathrm{D}}\right)$, where $T_{F}$ is the tetrahedron of $\mathcal{T}_{h}^{\mathrm{D}}$ having $F$ as a face, and

$$
\text { h.o.t }:=h_{T_{F}}^{4}\left\|\boldsymbol{u}_{\mathrm{D}}-\boldsymbol{u}_{h}^{\mathrm{D}}\right\|_{0, T_{F}}^{2}+h_{T_{F}}^{2}\left\|p_{\mathrm{D}}-p_{h}^{\mathrm{D}}\right\|_{0, T_{F}}^{2} \text {. }
$$

Proof Thanks to the fact that $\operatorname{curl}\left(\boldsymbol{f}_{\mathrm{D}}-\kappa_{\mathrm{D}}^{-1} \boldsymbol{u}_{\mathrm{D}}\right)=\operatorname{curl}\left(\nabla p_{\mathrm{D}}\right)=\mathbf{0}$ in $\Omega_{\mathrm{D}}$, (a) and (c) can be obtained applying (3.27) and (3.28), respectively, to $\zeta:=f_{\mathrm{D}}-\kappa_{\mathrm{D}}^{-1} \boldsymbol{u}_{\mathrm{D}}$ and $\zeta_{h}:=f_{\mathrm{D}}-\kappa_{\mathrm{D}}^{-1} \boldsymbol{u}_{h}^{\mathrm{D}}$. The remaining estimates follow analogously to the proofs of (b) and (d) in Lemma 20.

We next turn to the derivation of local efficiency estimates for the residual expressions defining $\widehat{\Theta}_{\mathrm{B}, T}^{2}$.

Lemma 22 There exist $c_{i}>0, i \in\{24,25,26\}$, independent of the meshsizes, such that

(a) $h_{T}^{2}\left\|\boldsymbol{\omega}_{h}^{\mathrm{B}}-\operatorname{curl} \boldsymbol{u}_{h}^{\mathrm{B}}\right\|_{0, T}^{2} \leq c_{24}\left\{\left\|\boldsymbol{u}_{\mathrm{B}}-\boldsymbol{u}_{h}^{\mathrm{B}}\right\|_{0, T}+h_{T}^{2}\left\|\boldsymbol{\omega}_{\mathrm{B}}-\boldsymbol{\omega}_{h}^{\mathrm{B}}\right\|_{0, T}\right\}$,

(b) $h_{F}\left\|\llbracket \boldsymbol{u}_{h}^{\mathrm{B}} \times \boldsymbol{n} \rrbracket\right\|_{0, F}^{2} \leq c_{25} \sum_{T \subseteq \omega_{F}}\left\{\left\|\boldsymbol{u}_{\mathrm{B}}-\boldsymbol{u}_{h}^{\mathrm{B}}\right\|_{0, T}^{2}+h_{T}^{2}\left\|\boldsymbol{\omega}_{\mathrm{B}}-\boldsymbol{\omega}_{h}^{\mathrm{B}}\right\|_{0, T}^{2}\right\} \forall F \in$ $\mathcal{F}_{h}\left(\Omega_{\mathrm{B}}\right)$,

(c) $h_{F}\left\|\llbracket \omega_{h}^{\mathrm{B}} \cdot \boldsymbol{n} \rrbracket\right\|_{0, F}^{2} \leq c_{26}\left\|\boldsymbol{\omega}_{\mathrm{B}}-\boldsymbol{\omega}_{h}^{\mathrm{B}}\right\|_{0, \omega_{F}}^{2}$.

Proof Regarding (a), let us denote $\chi_{T}:=\omega_{h}^{\mathrm{B}}-\operatorname{curl} \boldsymbol{u}_{h}^{\mathrm{B}}$ in a generic $T \in \mathcal{T}_{h}$. Applying the first estimate of (3.25) to $\chi_{T}$, and then using that curl $\boldsymbol{u}_{\mathrm{B}}=\boldsymbol{\omega}_{\mathrm{B}}$ in $\Omega_{\mathrm{B}}$, we find that

$$
\begin{aligned}
\left\|\chi_{T}\right\|_{0, T}^{2} \leq & c_{1}\left\|\psi_{T}^{1 / 2} \chi_{T}\right\|_{0, T}^{2}=c_{1} \int_{T} \psi_{T} \chi_{T} \cdot \operatorname{curl}\left(\boldsymbol{u}_{\mathrm{B}}-\boldsymbol{u}_{h}^{\mathrm{B}}\right) \\
& -c_{1} \int_{T} \psi_{T} \chi_{T} \cdot\left(\omega_{\mathrm{B}}-\omega_{h}^{\mathrm{B}}\right) .
\end{aligned}
$$

Next, integrating by parts in the first term on the right hand side of the last identity, and recalling that $\psi_{T}$ vanishes on $\partial T$, we obtain

$$
\left\|\chi_{T}\right\|_{0, T}^{2} \leq-c_{1} \int_{T}\left(\boldsymbol{u}_{\mathrm{B}}-\boldsymbol{u}_{h}^{\mathrm{B}}\right) \cdot \operatorname{curl}\left(\psi_{T} \chi_{T}\right)-c_{1} \int_{T} \psi_{T} \chi_{T} \cdot\left(\boldsymbol{\omega}_{\mathrm{B}}-\boldsymbol{\omega}_{h}^{\mathrm{B}}\right) .
$$

Then, applying Cauchy-Schwarz inequality and (3.26), we deduce from (3.36) that

$$
\left\|\boldsymbol{\chi}_{T}\right\|_{0, T}^{2} \leq c\left\|\psi_{T} \chi_{T}\right\|_{0, T}\left\{h_{T}^{-1}\left\|\boldsymbol{u}_{\mathrm{B}}-\boldsymbol{u}_{h}^{\mathrm{B}}\right\|_{0, T}+\left\|\boldsymbol{\omega}_{\mathrm{B}}-\boldsymbol{\omega}_{h}^{\mathrm{B}}\right\|_{0, T}\right\} .
$$


In this way, using that $0 \leq \psi_{T} \leq 1$ in $T$, we get

$$
h_{T}\left\|\chi_{T}\right\|_{0, T} \leq c\left\{\left\|\boldsymbol{u}_{\mathrm{B}}-\boldsymbol{u}_{h}^{\mathrm{B}}\right\|_{0, T}+h_{T}\left\|\boldsymbol{\omega}_{\mathrm{B}}-\boldsymbol{\omega}_{h}^{\mathrm{B}}\right\|_{0, T}\right\},
$$

which gives (a). Estimate (b) can be derived by adapting the arguments in the proof of [2, Lemma 4.15]. Finally, since $\operatorname{div}\left(\boldsymbol{\omega}_{\mathrm{B}}\right)=\operatorname{div}\left(\operatorname{curl} \boldsymbol{u}_{\mathrm{B}}\right)=0$ in $\Omega_{\mathrm{B}}$, for the derivation of (c), it suffices to apply (3.30) to $\zeta:=\omega_{\mathrm{B}}$ and $\zeta_{h}:=\omega_{h}^{\mathrm{B}}$.

We end this section by observing that the term $h_{F}\left\|\lambda-\lambda_{h}\right\|_{0, F}^{2}$ appearing in Lemma 17 (items (a) and (b)), is bounded as follows:

$$
\sum_{F \in \mathcal{F}_{h}(\Sigma)} h_{F}\left\|\lambda-\lambda_{h}\right\|_{0, F}^{2} \leq h\left\|\lambda-\lambda_{h}\right\|_{0, \Sigma}^{2} \leq C h\left\|\lambda-\lambda_{h}\right\|_{1 / 2, \Sigma}^{2}
$$

Therefore the efficiency of $\Theta$ is a direct consequence of (3.24) and Lemmas 17-21.

\section{Numerical results}

In this section we provide two computational tests aimed at illustrating the properties of the estimator $\Theta$ introduced in Sect. 3.2. All linear systems are solved with the distributed multifrontal direct solver MUMPS.

Example 1 For our first test we design a mesh convergence example using two sets of closed-form solutions, and performing uniform and adaptive mesh refinement. The Darcy and Brinkman sub-domains consist of two boxes $\Omega_{\mathrm{D}}=(-0.5,0.5)^{3}, \Omega_{\mathrm{B}}=$ $(-0.125,0.125)^{2} \times(-0.4,0.4)$. The model parameters are $\kappa_{\mathrm{B}}^{-1}=10, \kappa_{\mathrm{D}}^{-1}=50$, $v=0.01$. The convergence of the method is assessed by computing errors between the following manufactured smooth exact solutions

$$
\begin{aligned}
& \boldsymbol{\omega}_{\mathrm{B}}\left(x_{1}, x_{2}, x_{3}\right)=\left(\begin{array}{c}
-3 \pi \sin \left(\pi x_{1}\right) \cos \left(\pi x_{2}\right) \cos \left(\pi x_{3}\right) \\
3 \pi \cos \left(\pi x_{1}\right) \sin \left(\pi x_{2}\right) \cos \left(\pi x_{3}\right) \\
0
\end{array}\right), \\
& \boldsymbol{u}\left(x_{1}, x_{2}, x_{3}\right)=\left(\begin{array}{c}
\cos \left(\pi x_{1}\right) \sin \left(\pi x_{2}\right) \sin \left(\pi x_{3}\right) \\
\sin \left(\pi x_{1}\right) \cos \left(\pi x_{2}\right) \sin \left(\pi x_{3}\right) \\
-2 \sin \left(\pi x_{1}\right) \sin \left(\pi x_{2}\right) \cos \left(\pi x_{3}\right)
\end{array}\right), \\
& p\left(x_{1}, x_{2}, x_{3}\right)=\sin \left(\pi x_{1}\right) \sin \left(\pi x_{2}\right) \sin \left(\pi x_{3}\right), \\
& \boldsymbol{u}_{\mathrm{B}}=\left.\boldsymbol{u}\right|_{\Omega_{\mathrm{B}}}, \boldsymbol{u}_{\mathrm{D}}=\boldsymbol{u}_{\Omega_{\mathrm{D}}}, \quad p_{\mathrm{B}}=\left.p\right|_{\Omega_{\mathrm{B}}}, \quad p_{\mathrm{D}}=\left.p\right|_{\Omega_{\mathrm{D}}}, \lambda=\left.p\right|_{\Sigma},
\end{aligned}
$$

and their finite element approximations using a $\mathbb{R T}_{0}-\mathbb{N D}_{1}-\mathbb{R T}_{0}-\mathbf{P}_{0}-\mathbf{P}_{0}-\mathbf{P}_{1}$ family.

The domains are discretised into a series of nested uniform triangulations, where errors, experimental convergence rates, and effectivity indexes will be defined as 


$$
\begin{aligned}
e\left(\boldsymbol{u}_{\mathrm{B}}\right):= & \left\|\boldsymbol{u}_{\mathrm{B}}-\boldsymbol{u}_{\mathrm{B} h}\right\|_{\mathrm{div}, \Omega_{\mathrm{B}}}, e\left(\boldsymbol{\omega}_{\mathrm{B}}\right):=\left\|\boldsymbol{\omega}_{\mathrm{B}}-\boldsymbol{\omega}_{\mathrm{B} h}\right\|_{\text {curl }, \Omega_{\mathrm{B}}}, \\
e\left(\boldsymbol{u}_{\mathrm{D}}\right):= & \left\|\boldsymbol{u}_{\mathrm{D}}-\boldsymbol{u}_{\mathrm{D} h}\right\|_{\operatorname{div}, \Omega_{\mathrm{D}}}, e\left(p_{\mathrm{B}}\right):=\left\|p_{\mathrm{B}}-p_{\mathrm{B} h}\right\|_{0, \Omega_{\mathrm{B}}}, \\
e\left(p_{\mathrm{D}}\right):= & \left\|p_{\mathrm{D}}-p_{\mathrm{D} h}\right\|_{0, \Omega_{\mathrm{D}}}, e(\lambda):=\left\|\lambda-\lambda_{h}\right\|_{1 / 2, \Sigma}, \\
r(\cdot):= & \frac{\log (e(\cdot) / \hat{e}(\cdot))}{-\frac{1}{2} \log (N / \hat{N})}, e:=\left\{\left[e\left(\boldsymbol{u}_{\mathrm{B}}\right)\right]^{2}+\left[e\left(\boldsymbol{\omega}_{\mathrm{B}}\right)\right]^{2}+\left[e\left(\boldsymbol{u}_{\mathrm{D}}\right)\right]^{2}\right. \\
& \left.+\left[e\left(p_{\mathrm{B}}\right)\right]^{2}+\left[e\left(p_{\mathrm{D}}\right)\right]^{2}+[e(\lambda)]^{2}\right\}^{1 / 2}, \quad \operatorname{eff}(\Theta):=\frac{e}{\Theta},
\end{aligned}
$$

with $e$ and $\hat{e}$ denoting errors associated to two consecutive meshes of sizes $h$ and $\hat{h}$, and being associated to methods having $N$ and $\hat{N}$ degrees of freedom, respectively. The first two parts of Table 1 show optimal convergence for all fields under either adaptive or uniform mesh refinement.

Secondly, we regard the same domains but manufacture an exact pressure that is singular near one wall of $\Omega_{\mathrm{D}}$ (the singularity being located at $\left(x_{a}, x_{b}, x_{c}\right)=$ $(0,0,-0.55))$ :

$$
p=\left(\left(x_{1}-x_{a}\right)^{2}+\left(x_{2}-x_{b}\right)^{2}+\left(x_{3}-x_{c}\right)^{2}\right)^{-1}+\sin \left(\pi x_{1}\right) \sin \left(\pi x_{2}\right) \sin \left(\pi x_{3}\right) .
$$

We expect that the convergence is hindered by the lower regularity of the exact solution. This is indeed evidenced in the third block of Table 1, where we see an oscillating effectivity index and a very low convergence, especially so for the Darcy pressure and the Lagrange multiplier. The optimal character of the error decay is however restored when we use an adaptive mesh refinement strategy (see the last section of the table). We also confirm that the error indicator $\Theta$ performs well even if the fluid viscosity $v$ has a considerable variation (see Table 2). Intermediate adapted meshes and some components of the approximate solution are displayed in Fig. 1.

Example 2 Next we turn to the simulation of the flow behaviour within a composite domain $\Omega=(0,2) \times(0,0.2) \times(0.75)$. A smooth interface exists between the Darcy and Brinkman subdomains, where the Brinkman part is on top (see related test cases in $[1,4,10,14])$. For this problem we assume a uniform current flow on the $x_{1}$-direction and the presence of gravity, so $f_{\mathrm{B}}=f_{\mathrm{D}}=(0.25,0,-0.1)^{T}$. In addition, we take a dimensional parameters specified as $\kappa_{\mathrm{B}}^{-1}=1, v=0.01$, and $\kappa_{\mathrm{D}}^{-1}=8+800 \eta\left(x_{1}, x_{2}, x_{3}\right)$, where $\eta$ is the sum of characteristic functions on 20 balls of radius $1 \mathrm{E}-4$, located randomly in $\Omega_{\mathrm{D}}$ and representing obstacles of much lower permeability.

The boundary conditions are set as follows: on the face $x_{1}=0$ we impose a unitary normal inflow velocity $\boldsymbol{u}_{\mathrm{B}} \cdot \boldsymbol{n}=1$, on the bottom and top faces we set slip velocity conditions $\boldsymbol{u}_{\mathrm{B}} \cdot \boldsymbol{n}=0$ and $\boldsymbol{u}_{\mathrm{D}} \cdot \boldsymbol{n}=0$ (for the Brinkman and Darcy boundaries, respectively), and on the remaining parts of the boundary we do not force velocity nor pressure. On the interface we impose zero tangential vorticity and the transmission conditions analyzed in the paper. We now use the method based on the $\mathbb{R T}_{1}-\mathbb{N D}_{2}-$ $\mathbb{R} \mathbb{T}_{1}-\mathbf{P}_{1}-\mathbf{P}_{1}-\mathbf{P}_{2}$ family, and a penalisation approach is used to impose zero-mean value of the Brinkman pressure. In Fig. 2 we present the sketch of the domains and 


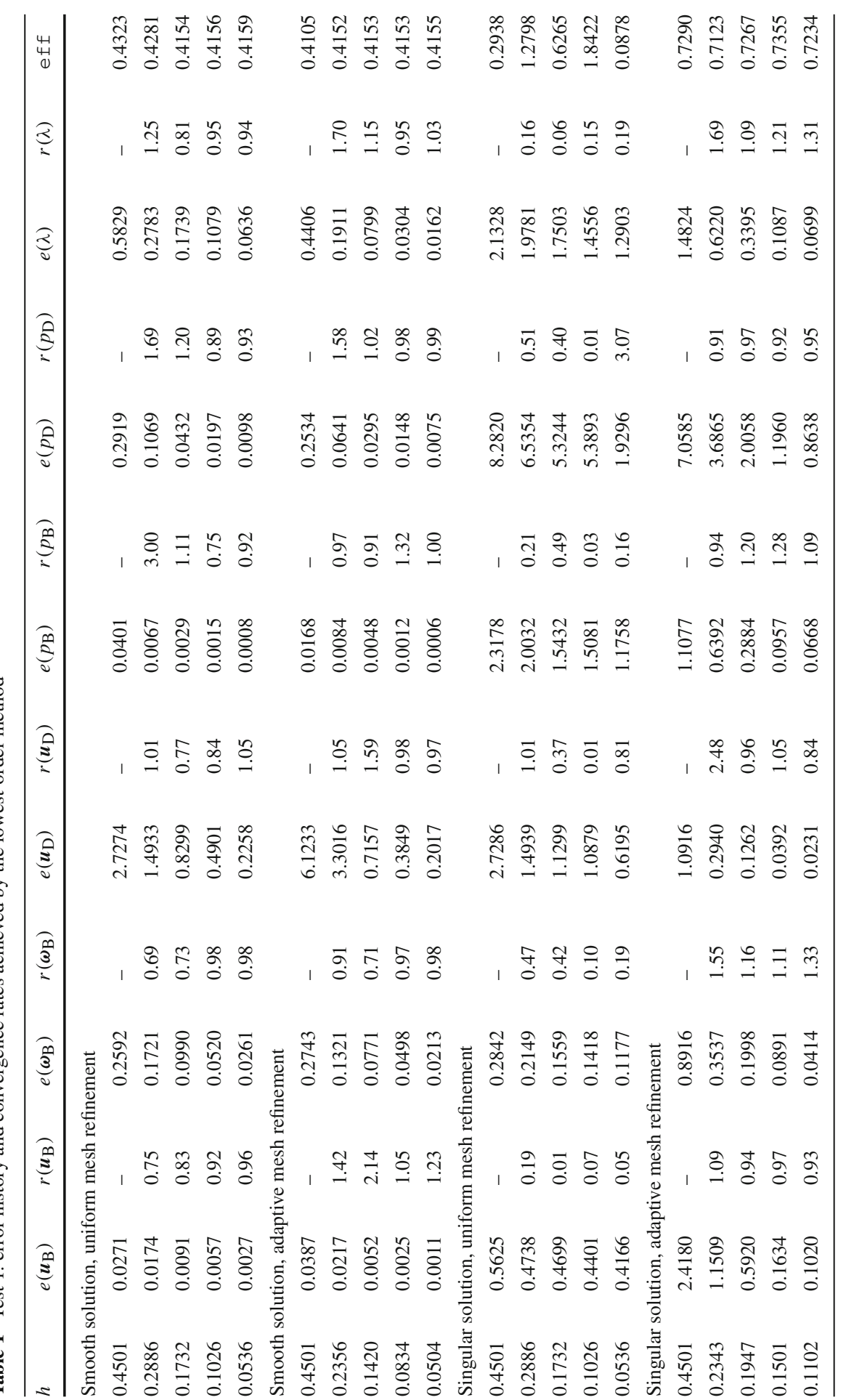


Table 2 Test 1: total error decay and effectivity indexes achieved by the lowest-order method for different values of the fluid viscosity

\begin{tabular}{|c|c|c|c|c|c|c|c|}
\hline \multicolumn{2}{|c|}{$\begin{array}{l}\text { Smooth solution, } \\
\text { uniform refinement }\end{array}$} & \multicolumn{2}{|c|}{$\begin{array}{l}\text { Smooth solution, } \\
\text { adaptive refinement }\end{array}$} & \multicolumn{2}{|c|}{$\begin{array}{l}\text { Singular solution, } \\
\text { uniform refinement }\end{array}$} & \multicolumn{2}{|c|}{$\begin{array}{l}\text { Singular solution, } \\
\text { adaptive refinement }\end{array}$} \\
\hline e & eff & e & eff & e & eff & e & eff \\
\hline \multicolumn{8}{|c|}{$v=0.01$} \\
\hline 2.8166 & 0.4323 & 6.1506 & 0.4105 & 9.2927 & 0.2938 & 7.8154 & 0.7290 \\
\hline 1.5326 & 0.4281 & 3.3105 & 0.4152 & 7.2897 & 1.2798 & 3.9902 & 0.7123 \\
\hline 0.8548 & 0.4154 & 0.7249 & 0.4153 & 5.9427 & 0.6265 & 2.1513 & 0.7267 \\
\hline 0.5049 & 0.4156 & 0.3896 & 0.4153 & 5.9021 & 1.8422 & 1.2197 & 0.7355 \\
\hline 0.2363 & 0.4159 & 0.2036 & 0.4155 & 2.7096 & 0.0878 & 0.8764 & 0.7234 \\
\hline \multicolumn{8}{|l|}{$v=10$} \\
\hline 2.8043 & 0.4149 & 6.1288 & 0.4092 & 9.8591 & 0.3372 & 6.9503 & 0.7248 \\
\hline 1.4311 & 0.4291 & 3.4192 & 0.4083 & 6.0251 & 2.6394 & 3.7943 & 0.7496 \\
\hline 0.7847 & 0.4107 & 0.7504 & 0.4107 & 5.5590 & 0.8520 & 2.0643 & 0.7250 \\
\hline 0.5152 & 0.4197 & 0.4121 & 0.4113 & 4.4921 & 2.4083 & 1.0251 & 0.7394 \\
\hline 0.2520 & 0.4103 & 0.2013 & 0.4171 & 3.0435 & 1.3107 & 0.8184 & 0.7254 \\
\hline
\end{tabular}

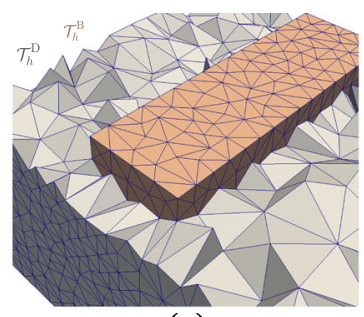

(a)

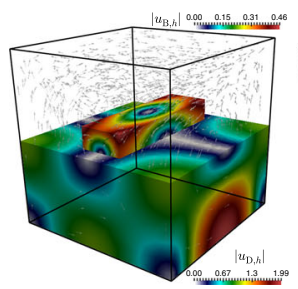

(c)

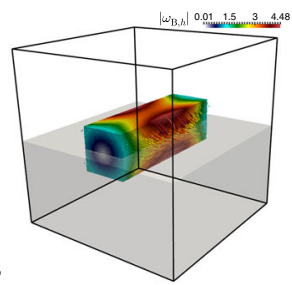

(d)

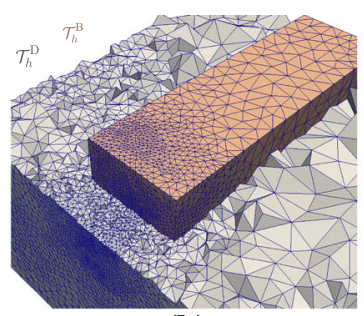

(b)

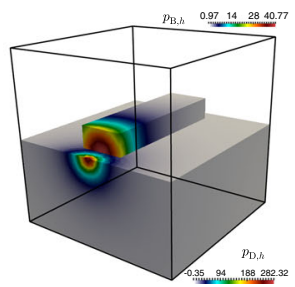

(e)

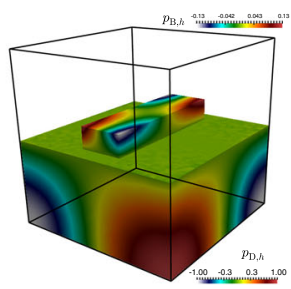

(f)

Fig. 1 Test 1 a, b zoom on the interface of two intermediate adapted meshes for the case of high gradient pressure, generated with the adaptive method; c, d global velocity and Brinkman vorticity obtained with a uniform refinement; and $\mathbf{e}, \mathbf{f}$ global pressure computed with the adaptive method in the case of a high gradient, and smooth pressure profile, respectively. In all figures we represent only a part of $\Omega_{\mathrm{D}}$, for visualisation purposes

interface, the obtained approximate solutions and snapshots of two adaptive meshes produced following the a posteriori error estimator. All fields are well-resolved, even with coarse grids. 


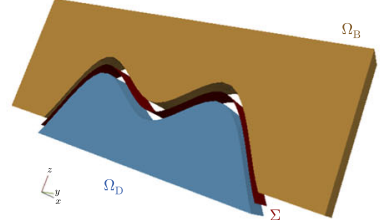

(a)

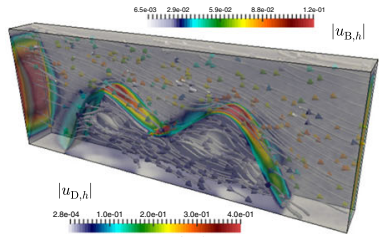

(d)

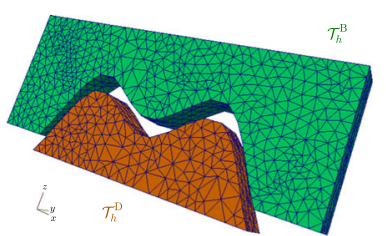

(b)

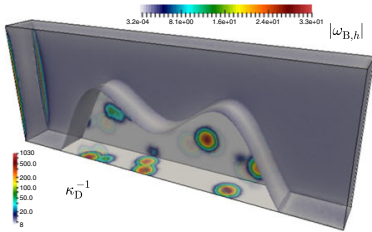

(e)

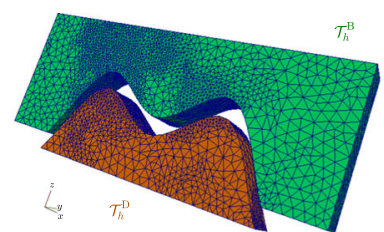

(c)

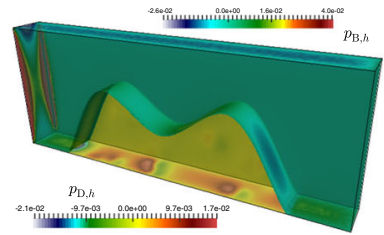

(f)

Fig. 2 Test 2 a domains sketch, b, c two intermediate adapted meshes, d global velocity, e Brinkman vorticity and Darcy inverse permeability, $\mathbf{f}$ global pressure

Acknowledgements This work was partially supported by CONICYT-Chile through BASAL project CMM, Universidad de Chile, and project Anillo ACT1118 (ANANUM); by the Ministery of Education through the project REDOC.CTA of the Graduate School, Universidad de Concepción; by Centro de Investigación en Ingeniería Matemática ( $\left.\mathrm{CI}^{2} \mathrm{MA}\right)$, Universidad de Concepción; by Vicerrectoría de Investigación, project 540-B7-233, Sede de Occidente, Universidad de Costa Rica; and by the EPSRC through the research grant EP/R00207X/1.

Open Access This article is distributed under the terms of the Creative Commons Attribution 4.0 International License (http://creativecommons.org/licenses/by/4.0/), which permits unrestricted use, distribution, and reproduction in any medium, provided you give appropriate credit to the original author(s) and the source, provide a link to the Creative Commons license, and indicate if changes were made.

\section{References}

1. Álvarez, M., Gatica, G.N., Ruiz-Baier, R.: Analysis of a vorticity-based fully-mixed formulation for the 3D Brinkman-Darcy problem. Comput. Methods Appl. Mech. Eng. 307, 68-95 (2016)

2. Anaya, V., Gatica, G.N., Mora, D., Ruiz-Baier, R.: An augmented velocity-vorticity-pressure formulation for the Brinkman problem. Int. J. Numer. Methods Fluids 79(3), 109-137 (2015)

3. Anaya, V., Mora, D., Oyarzúa, R., Ruiz-Baier, R.: A priori and a posteriori error analysis of a mixed scheme for the Brinkman problem. Numer. Math. 133(4), 781-817 (2016)

4. Anaya, V., Mora, D., Reales, C., Ruiz-Baier, R.: A vorticity-pressure finite element formulation for the Brinkman-Darcy coupled problem. Preprint 2017-13, Centro de Investigación en Ingeniería Matemática (CI $\left.{ }^{2} \mathrm{MA}\right)$. Universidad de Concepción, Chile, (2017). http://www.ci2ma.udec.cl

5. Anaya, V., Mora, D., Ruiz-Baier, R.: Pure vorticity formulation and Galerkin discretization for the Brinkman equations. IMA J. Numer. Anal. (2017). doi:10.1093/imanum/drw056

6. Babuška, I., Gatica, G.N.: A residual-based a posteriori error estimator for the Stokes-Darcy coupled problem. SIAM J. Numer. Anal. 48(2), 498-523 (2010)

7. Barrios, T.P., Gatica, G.N., González, M., Heuer, N.: A residual based a posteriori error estimator for an augmented mixed finite element method in linear elasticity. M2AN Math. Model. Numer. Anal. 40(5), 843-869 (2006)

8. Bernardi, C., Hecht, F.: Quelques proriétés d'approximation des éléments finis de Nédélec, application à l'analyse a posteriori. C. R. Acad. Sci. Paris Ser. I 344, 461-466 (2007)

9. Bernardi, C., Chacón, T., Hecht, F., Mghazli, Z.: Mortar finite element discretization of a model coupling Darcy and Stokes equations. ESAIM Math. Model. Numer. Anal. 42, 375-410 (2008) 
10. Bernardi, C., Hecht, F., Nouri, F.Z.: A new finite-element discretization of the Stokes problem coupled with the Darcy equations. IMA J. Numer. Anal. 30, 61-93 (2010)

11. Bernardi, C., Hecht, F., Pironneau, O.: Coupling Darcy and Stokes equations for porous media with cracks. ESAIM Math. Model. Numer. Anal. 39, 7-35 (2005)

12. Brezzi, F., Fortin, M.: Mixed and Hybrid Finite Element Methods. Springer, New York (1991)

13. Buffa, A., Costabel, M., Sheen, D.: On traces for $\mathbf{H}(\mathbf{c u r l}, \Omega)$ in Lipschitz domains. J. Math. Anal. Appl. 276(2), 845-867 (2002)

14. Camaño, J., Gatica, G.N., Oyarzúa, R., Ruiz-Baier, R., Venegas, P.: New fully-mixed finite element methods for the Stokes-Darcy coupling. Comput. Methods Appl. Mech. Eng. 295, 362-395 (2015)

15. Carstensen, C.: A posteriori error estimate for the mixed finite element method. Math. Comput. 66(218), 465-476 (1997)

16. Caucao, S., Gatica, G.N., Oyarzúa, R.: A posteriori error analyis of a fully-mixed formulation for the Navier-Stokes/Darcy coupled problem with nonlinear viscosity. Comput. Methods Appl. Mech. Eng. 315, 943-971 (2017)

17. Caucao, S., Gatica, G.N., Oyarzúa, R., Šebestová, I.: A fully-mixed finite element method for the Navier-Stokes/Darcy coupled problem with nonlinear viscosity. J. Numer. Math. 25(2), 55-88 (2017)

18. Chesnel, L., Ciarlet, P.: T-coercivity and continuous Galerkin methods: application to transmission problems with sign changing coefficients. Numer. Math. 124(1), 1-129 (2013)

19. Ciarlet, P.: The Finite Element Method for Elliptic Problems. North-Holland, Amsterdam (1978)

20. Clément, P.: Approximation by finite element functions using local regularization. RAIRO Modél. Math. Anal. Numer. 9(2), 77-84 (1975)

21. Cochez-Dhondt, S., Nicaise, S.: Robust a posteriori error estimation for the Maxwell equations. Comput. Methods Appl. Mech. Eng. 197, 2583-2595 (2007)

22. Gatica, G.N.: A Simple Introduction to the Mixed Finite Element Method. Theory and Applications. Springer, Berlin (2014)

23. Gatica, G.N.: A note on stable Helmholtz decompositions in 3D. Preprint 2016-03, Centro de Investigación en Ingeniería Matemática ( $\left.\mathrm{CI}^{2} \mathrm{MA}\right)$. Universidad de Concepción, Chile, (2016). http://www. ci2ma.udec.cl

24. Gatica, G.N., Gatica, L.F., Sequeira, F.A.: A priori and a posteriori error analyses of a pseudostressbased mixed formulation for linear elasticity. Comput. Math. Appl. 71(2), 585-614 (2016)

25. Gatica, G.N., Hsiao, G.C., Meddahi, S.: A residual-based a posteriori error estimator for a two dimensional fluid-solid interaction problem. Numer. Math. 114(1), 63-106 (2010)

26. Gatica, G.N., Márquez, A., Meddahi, S.: Analysis of the coupling of Lagrange and Arnold-FalkWinther finite elements for a fluid-solid interaction problem in 3D. SIAM J. Numer. Anal. 50(3), 1648-1674 (2012)

27. Gatica, G.N., Oyarzúa, R., Sayas, F.J.: A residual-based a posteriori error estimator for a fully-mixed formulation of the Stokes-Darcy coupled problem. Comput. Methods Appl. Mech. Eng. 200(21-22), 1877-1891 (2011)

28. Gatica, G.N., Ruiz-Baier, R., Tierra, G.: A posteriori error analysis of an augmented mixed method for the Navier-Stokes equations with nonlinear viscosity. Comput. Math. Appl. 72(9), 2289-2310 (2016)

29. Gatica, G.N., Ruiz-Baier, R., Tierra, G.: A mixed finite element method for Darcy's equations with pressure dependent porosity. Math. Comput. 85(297), 1-33 (2016)

30. Girault, V., Raviart, P.A.: Finite Element Methods for Navier-Stokes Equations. Theory and Algorithms. Springer, Berlin (1986)

31. Pasciak, J., Zhao, J.: Overlapping Schwarz methods in H(curl) on polyhedral domains. J. Numer. Math. 10, 221-234 (2002)

32. Verfürth, R.: A posteriori error estimation and adaptive-mesh-refinement techniques. J. Comput. Appl. Math. 50(1-3), 67-83 (1994)

33. Verfürth, R.: A Review of A Posteriori Error Estimation and Adaptive-Mesh-Refinement Techniques. Wiley-Teubner, Chichester (1996)

34. Verfürth, R.: A Posteriori Error Estimation Techniques for Finite Element Methods. Numerical Mathematics and Scientific Computation. Oxford University Press, Oxford (2013) 